T. Shintani

Nagoya Math. J.

Vol. 58 (1975), 83-126

\title{
ON CONSTRUCTION OF HOLOMORPHIC CUSP FORMS OF HALF INTEGRAL WEIGHT
}

\author{
TAKURO SHINTANI*
}

\section{Introduction}

In [10], G.Shimura gave a method of constructing holomorphic cusp forms of even integral weight from given forms of half integral weight. In this paper, we try to present an inverse construction. To state our main result, some notational preliminaries are necessary. We denote by $\mathfrak{S}_{\mathfrak{S}}$ the complex upper half plane. Let $x(u, v)=x_{1} u^{2}+x_{2} u v+x_{3} v^{2}$ be an integral binary quadratic form with positive discriminant $d_{x}=x_{2}{ }^{2}-4 x_{1} x_{3}$. If $d_{x}=m^{2}(m>0)$ is a square, we denote by $C(x)$ the geodesic line with respect to the Poincaré metric on $\mathscr{F}$ from $\left(x_{2}+m\right) / 2 x_{3}$ to $\left(x_{2}-m\right) / 2 x_{3}$ (if $x_{3}=0$, we understand $C(x)$ to be the geodesic line from $+i \infty$ (resp. $\left.x_{1} / x_{2}\right)$ to $x_{1} / x_{2}($ resp. $+i \infty)$ for $x_{2}>0$ (resp. $\left.x_{2}<0\right)$ ). If $d_{x}$ is not a square and if $x_{1}, x_{2}$ and $x_{3}$ have no non-trivial common divisor, let $t_{x}+u_{x} \sqrt{d_{x}}>1$ be the smallest half-integer solution of the Pell-equation $t^{2}-u^{2} d_{x}=1$ and set $\gamma_{x}=\left(\begin{array}{cc}t_{x}-x_{2} u & 2 x_{1} u \\ -2 x_{3} u & t_{x}+x_{2} u\end{array}\right) \in S L_{2}(Z)$. We denote by $C(x)$ any rectifiable curve in $\mathfrak{S}$ from $w$ to $\gamma_{x} \cdot w$, where $w$ is any point on $\mathfrak{S}_{\mathrm{g}}$. Finally if $x_{1}, x_{2}$ and $x_{3}$ have the greatest common divisor $t>1$, we put $C(x)=$ $C(x / t)$.

Now, let $f(z)$ be a holomorphic cusp form on the upper half plane which satisfies

$$
f(\gamma \cdot z)=\chi^{2}(d)(c z+d)^{2 k} f(z)
$$

for any $r=\left(\begin{array}{ll}a & b \\ c & d\end{array}\right) \in \Gamma_{0}(N)$, where $\chi$ is a character modulo $N$ and $\Gamma_{0}(N)$ $=\left\{\left[\begin{array}{ll}a & b \\ c & d\end{array}\right] \in S L_{2}(Z) \mid c \equiv 0(\bmod . N)\right\} . \quad$ Two integral binary quadratic

Received November 11, 1974.

* During the preparation of this work the author was supported in part by National Science Foundation Grant GP-36418 X. 
forms $x^{1}$ and $x^{2}$ are said to be $\Gamma_{0}(N)$-equivalent if $x^{1}(u, v)=x^{2}((u, v) \gamma)$ for a suitable $\gamma \in \Gamma_{0}(N)$.

For simplicity we assume $N$ to be odd and put

$$
\begin{aligned}
\theta(f, z)= & \sum_{x} \chi\left(x_{1}\right) \exp \left(\frac{2 \pi i}{N} d_{x} z\right) \\
& \times \int_{C(x)} f(w)\left(x_{1}-x_{2} w+x_{3} w^{2}\right)^{k-1} d w,
\end{aligned}
$$

where the summation is taken over all $\Gamma_{0}(N)$-equivalence classes of integral binary quadratic forms $x(u, v)=x_{1} u^{2}+x_{2} u v+x_{3} v^{2}$ with positive discriminant $d_{x}=x_{2}^{2}-4 x_{1} x_{3}$ and with $x_{2}$ and $x_{3}$ both divisible by $N$ and with $x_{1}$ prime to $N$. Then our Theorem 2 asserts that $\theta(f, z)$ is a holomorphic cusp form of weight $k+1 / 2$ which satisfies

$$
\theta(f, \gamma \cdot z)=\chi^{\prime}(d) j(\gamma, z)^{2 k+1} \theta(f, z)
$$

for any $\gamma=\left(\begin{array}{ll}a & b \\ c & d\end{array}\right) \in \Gamma_{0}(4 N)$, where $j(\gamma, z)$ is an automorphic factor given by $(1.9 \mathrm{~b})$ of [10] and $\chi^{\prime}$ is a character modulo $4 N$ given by $\chi^{\prime}(d)=$ $\chi(d)(-1 / d)^{x}\left(\frac{N}{d}\right)$. Moreover, the mapping $f \mapsto \theta(z, f)$ commutes with the action of Hecke operators. It should also be mentioned that the integral representation (0.1) gives geometric interpretation of the Fourier coefficients of cusp forms of half-integral weight (cf. the question (B) in p. 478 of [10], and the discussion of $\S 3$ ).

The series which appear in the right hand side of $(0.1)$ is a kind of theta series. Theta series of similar nature were previously studied in Siegel [12], Maass [7], [8], Weil [13] and in Shalika-Tanaka [9].

This paper consists of three sections. In the first section, which is of expository nature, we derive certain transformation formulas for theta series by means of Weil theory [13]. We prove our main theorem in the second section and discuss a few numerical examples in the last section. The author wishes to express his hearty thanks to Professor G. Shimura, for his valuable suggestions and warm encouragement.

\section{Notations}

1. We denote, as usual, by $Z, Q, R$ and $C$ the ring of rational integers, the rational number field, the real number field, and the complex number field. Also we put $T=\{z \in C|| z \mid=1\}$. For $z \in C$, we put $e[z]$ $=\exp 2 \pi \sqrt{-1} z$ and define $\sqrt{z}=z^{1 / 2}$ so that $-(\pi / 2)<\arg z^{1 / 2} \leqslant \pi / 2$. Further 
we put $z^{k / 2}=\left(z^{1 / 2}\right)^{k}$ for every $k \in Z$. For a non zero real number $x$, we set $\operatorname{sgn} x=x /|x|$. For a finite dimensional real vector space $V$, we denote by $\mathscr{S}(V)$ the space of rapidly decreasing functions on $V$. For a symmetric matrix $Q$ of size $n$ we put $Q(x)={ }^{t} x Q x\left(x \in \boldsymbol{R}^{n}\right)$.

2. In this paper "quadratic residue symbol" $\left(\frac{a}{b}\right)$ has the same meaning as in [10].

\section{§1. Theta series}

1. In the following three paragraphs, we summarize some results of Weil [13], in a form convenient for later applications. Let $V$ be an $n$-dimensional real vector space and let $V^{*}$ be the vector space dual to $V$. Denote by $B$ a bilinear form on $\left(V \times V^{*}\right) \times\left(V \times V^{*}\right)$ given by $B\left(z_{1}, z_{2}\right)$ $=\left(v_{1}, v_{2}^{*}\right)$ for $z_{1}=\left(v_{1}, v_{1}^{*}\right)$ and $z_{2}=\left(v_{2}, v_{2}^{*}\right)$. Let $A(V)$ be a Lie group with underlying manifold $V \times V^{*} \times T$ whose law of composition is given by

$$
\begin{aligned}
(z, t)\left(z^{\prime}, t^{\prime}\right)= & \left(z+z^{\prime}, t t^{\prime} e\left[B\left(z, z^{\prime}\right)\right]\right) \\
& \left(z, z^{\prime} \in V \times V^{*}, \quad t, t^{\prime} \in T\right) .
\end{aligned}
$$

We fix a Euclidean measure $d x$ on $V$ and denote by $d x^{*}$ the Euclidean measure which is dual to $d x$. Namely, the Fourier transform $f^{*}\left(x^{*}\right) \mapsto$ $\int_{V^{*}} f^{*}\left(x^{*}\right) e\left[\left(x, x^{*}\right)\right] d x^{*}$ gives an isometric mapping from $L^{2}\left(V^{*}, d x^{*}\right)$ onto $L^{2}(V, d x)$. We denote by $U$ a unitary representation of $A(V)$ on $L^{2}(V)$ given by $\{U(z, t) f\}(x)=t e\left[\left(x, v^{*}\right)\right] f(x+v)\left(x \in V, z=\left(v, v^{*}\right) \in V \times V^{*}, t \in T\right)$. Then $U$ is irreducible and $\mathscr{S}(V)$, the space of rapidly decreasing functions on $V$, is a dense invariant subspace of $L^{2}(V)$. A linear transformation of $V \times V^{*}$ is said to be symplectic if it leaves the alternating form $A\left(z_{1}, z_{2}\right)=B\left(z_{1}, z_{2}\right)-B\left(z_{2}, z_{1}\right)$ invariant. We denote by $\operatorname{Sp}\left(V \times V^{*}\right)$ the group of symplectic linear transformations of $V \times V^{*}$. We write, for $\sigma \in \operatorname{Sp}\left(V, V^{*}\right)$ and for $z=\left(v, v^{*}\right) \in V \times V^{*}$,

$$
z \sigma=\left(v, v^{*}\right)\left(\begin{array}{ll}
a & b \\
c & d
\end{array}\right)=\left(v a+v^{*} c, v b+v^{*} d\right)
$$

where $a, b, c$ and $d$ are linear mappings from $V$ to $V$, from $V$ to $V^{*}$, from $V^{*}$ to $V$ and from $V^{*}$ to $V^{*}$ respectively. In the following we often identify $\sigma$ with the matrix $\left(\begin{array}{ll}a & b \\ c & d\end{array}\right)$. For $\sigma \in \operatorname{Sp}\left(V \times V^{*}\right)$ and 
$z \in V \times V^{*}$, set

$$
F_{\sigma}(z)=\exp \pi \sqrt{-1} B(z \sigma, z \sigma) / \exp \pi \sqrt{-1} B(z, z) .
$$

It is easy to see that

$$
\left\{\begin{array}{l}
F_{\sigma}\left(z+z^{\prime}\right)=F_{\sigma}(z) F_{\sigma}\left(z^{\prime}\right) e\left[B\left(z \sigma, z^{\prime} \sigma\right)-B\left(z, z^{\prime}\right)\right], \\
F_{\sigma \tau}(z)=F_{\tau}(z \sigma) F_{\sigma}(z) .
\end{array}\right.
$$

These equalities show that the group $\operatorname{Sp}\left(V \times V^{*}\right)$ acts on $A(V)$ as a group of automorphisms via the mapping:

$$
w \mapsto w^{\sigma}=\left(z \sigma, t F_{\sigma}(z)\right) \quad(w=(z, t) \in A(V)) .
$$

Set $U^{\sigma}(w)=U\left(w^{o}\right)\left(\sigma \in \operatorname{Sp}\left(V \times V^{*}\right), w \in A(V)\right)$, then $U^{\sigma}$ is an irreducible unitary representation of $A(V)$ which is equivalent to $U$. Namely, there exists a unitary operator $r(\sigma)$ on $L^{2}(V)$ which satisfies

$$
U\left(w^{\sigma}\right)=r(\sigma)^{-1} U(w) r(\sigma) \quad \text { for every } w \in A(V) \text {. }
$$

The operator $r(\sigma)$ is unique up to a multiplication by a complex number of modulus 1. Furthermore, the mapping $\sigma \mapsto r(\sigma)$ gives rise to a projective unitary representation of $\operatorname{Sp}\left(V \times V^{*}\right)$ on $L^{2}(V)$. In other words, for each pair $(\sigma, \tau)\left(\sigma, \tau \in \operatorname{Sp}\left(V \times V^{*}\right)\right)$, there exists a constant $c(\sigma, \tau)$ which satisfies

$$
r(\sigma \tau)=c(\sigma, \tau) r(\sigma) r(\tau)
$$

This projective unitary representation is called the Weil representation of $\operatorname{Sp}\left(V \times V^{*}\right)$. If the left lower entry of the matrix form of $\sigma$ is either non-singular or zero, we may normalize $r(\sigma)$ as follows:

$$
r(\sigma) f(v)=\left\{\begin{array}{l}
|c|^{1 / 2} \int_{V^{*}} F_{\sigma}\left(\left(v, v^{*}\right)\right) f\left(v a+v^{*} c\right) d v^{*} \quad(c \text { is non-singular }), \\
|a|^{1 / 2} e\left[\frac{1}{2}(v a, v b)\right] f(v a) \quad(c=0),
\end{array}\right.
$$

for $\sigma=\left(\begin{array}{ll}a & b \\ c & d\end{array}\right)$, where we set $d\left(x^{*} c\right)=|c| d^{*} x^{*}$ and $d(x a)=|a| d x$.

2. Let $L$ be a lattice in $V$ and $L^{*}$ be the lattice dual to $L$ in $V^{*}$. Take a sublattice $M^{*}$ of $L^{*}$ and let $M$ be the dual lattice of $M^{*}$ in $V$. For any character $\chi$ of $L \times M^{*}$, we denote by the same letter $\chi$ the character of a subgroup $L \times M^{*} \times T$ of $A(V)$ given by 


$$
\chi((z, t))=t \chi(z) \quad\left(\forall z \in L \times M^{*}\right) .
$$

There exists a $\left(v_{\chi}, v_{\chi}^{*}\right) \in V \times V^{*}$ which satisfies

$$
\chi\left(\lambda, \mu^{*}\right)=e\left[\left(v_{x}, \mu^{*}\right)-\left(\lambda, v_{x}^{*}\right)\right] \quad\left(\forall\left(\lambda, \mu^{*}\right) \in L \times M^{*}\right) .
$$

The mapping $\chi \rightarrow\left(v_{x}, v_{x}^{*}\right)$ establishes an isomorphism between the character group of $L \times M^{*}$ and the additive group $V / M \times V^{*} / L^{*}$. For each $\mu \in M / L$, we denote by $\chi(\mu)$ the character of $L \times L^{*}$ which corresponds to $\left(v_{x}+\mu, v_{x}^{*}\right)$ of $V / L \times V / L^{*}$. Any extension of $\chi$ to a character of $L \times L^{*}$ coincides with $\chi(\mu)$ for a suitable $\mu \in M / L$. We denote by $T_{\chi}\left(L \times M^{*}\right)$ a unitary representation of $A(V)$ induced from the character $\chi$ of $L \times M^{*} \times T$. The representation space $\Theta_{\chi}\left(L \times M^{*}\right)$ of $T_{\chi}\left(L \times M^{*}\right)$ is the Hilbert space of measurable functions on $V \times V^{*}$ which satisfy the following conditions (1.6)

$$
\left\{\begin{array}{l}
e[B(\lambda, z)] \theta(\lambda+z)=\chi(\lambda) \theta(z) \quad\left(\forall \lambda \in L \times M^{*}, z \in V \times V^{*}\right), \\
\|\theta\|^{2}=\int_{V / L \times V^{*} / M^{*}}\left|\theta\left(x, x^{*}\right)\right|^{2} d x d x^{*}<\infty .
\end{array}\right.
$$

The representation $T_{\chi}\left(L \times M^{*}\right)$ is given by

$$
T_{\chi}\left(L, M^{*}\right)((w, t)) \theta(z)=t e[B(z, w)] \theta(z+w) .
$$

It is easy to see that the space $\Theta_{x(\mu)}\left(L \times L^{*}\right)$ is, for each $\mu \in M / L$, a closed invariant subspace of $\Theta_{x}\left(L \times M^{*}\right)$. Moreover, we have the following orthogonal direct sum decomposition of $\Theta_{x}\left(L \times M^{*}\right)$.

$$
\Theta_{\chi}\left(L \times M^{*}\right)=\bigoplus_{\mu \in M / L} \Theta_{\chi(\mu)}\left(L \times L^{*}\right) .
$$

For abbreviation, we write

$$
\Theta_{\chi}=\Theta_{\chi}\left(L \times M^{*}\right), \quad \Theta_{\chi(\mu)}=\Theta_{\chi(\mu)}\left(L \times L^{*}\right) \text { and } T_{\chi}=T_{\chi}\left(L \times M^{*}\right) .
$$

We will show that the unitary representation $\left(T_{x}, \Theta_{\chi(\mu)}\right)$ of $A(V)$ is equivalent to $\left(U, L^{2}(V)\right)\left({ }^{\forall} \mu \in M / L\right)$. For that purpose, set, for an $f \in \mathscr{S}(V)$,

$$
\begin{aligned}
\theta_{\chi(\mu)}(f)\left(x, x^{*}\right)= & {\sqrt{\operatorname{vol}\left(V^{*} / M^{*}\right)^{-1}}} \\
& \times \sum_{l \in L} e\left[\left(l+\mu+v_{\chi}, x^{*}\right)+\left(l, v_{\chi}^{*}\right)\right] f\left(x+l+\mu+v_{\chi}\right),
\end{aligned}
$$

where $\operatorname{vol}\left(V^{*} / M^{*}\right)=\int_{V^{*} / M^{*}} d x^{*}$.

We note that $\theta_{x(\mu)}(f)$ depends upon the choice of a representative for $\left(v_{x}+\mu\right) \in V / L$ in $V$. If we replace $\left(v_{z}+\mu\right)$ by $\left(v_{x}+\mu+l^{\prime}\right)\left(l^{\prime} \in L\right), \theta_{x^{(\mu)}}(f)$ 
is multiplied by $e\left[-\left(l^{\prime}, v_{x}^{*}\right)\right]$. In the following we choose representatives for $\left(v_{x}+\mu\right)(\mu \in M / L)$ and fix them once and for all. Then it is easy to see that $\theta_{\chi^{(\mu)}}(f)$ is a smooth function in $\Theta_{\chi^{(\mu)}}$ and that

$$
\left\{\begin{array}{l}
\theta_{\chi(\mu)}\{U(g) f\}=T_{\chi}(g) \theta_{\chi(\mu)}(f) \quad\left({ }^{\forall} g \in A(V)\right), \\
\left\|\theta_{\chi(\mu)}(f)\right\|^{2}=\|f\|^{2}=\int_{V}|f(x)|^{2} d x .
\end{array}\right.
$$

Conversely, for a smooth function $\theta$ in $\Theta_{\chi(\mu)}$, set

$$
f_{\theta}(x)=\sqrt{\operatorname{vol}\left(V^{*} / M^{*}\right)^{-1}} \int_{V^{*} / M^{*}} \theta\left(x-\mu-v_{x}, x^{*}\right) e\left[-\left(\mu+v_{x}, x^{*}\right)\right] d x^{*} .
$$

Then it is easy to see that $f_{\theta}$ is a rapidly decreasing function on $V$ and that $\theta_{\chi(\mu)}\left(f_{\theta}\right)=\theta$. Hence, $\theta_{\chi(\mu)}$ gives a norm preserving linear mapping from $\mathscr{S}(V)$ onto the space of smooth functions in $\Theta_{x(\mu)}$ which commutes with the action of $A(V)$.

Furthermore, the inverse of $\theta_{x(\mu)}$ is given by (1.10). Therefore, $\theta_{x(\mu)}$ is extended to a linear isometric mapping from $L_{2}(V)$ onto $\Theta_{\chi(u)}$ which establishes the equivalence of two unitary representations $\left(U, L^{2}(V)\right)$ and $\left(T_{\chi}, \Theta_{\chi(\mu)}\right)$. Since $\left(U, L^{2}(V)\right)$ is irreducible and $\left(T_{x}, \Theta_{\chi}\right)$ is a direct sum of $\left(T_{\chi}, \Theta_{\chi(\mu)}\right)(\mu \in M / L)$, any bounded linear mapping of $L^{2}(V)$ into $\Theta_{\chi}$ is a linear combinations of $\theta_{x(\mu)}(\mu \in M / L)$ if it commutes with the action of $A(V)$. Put

$$
\theta(f, \chi(\mu))=\theta_{\chi(\mu)}(f)(0,0) .
$$

3. Denote by $\operatorname{Sp}\left(L \times M^{*}\right)$ the subgroup of $\operatorname{Sp}\left(V \times V^{*}\right)$ consisting of linear transformations which leave the lattice $L \times M^{*}$ invariant. For a character $\chi$ of $L \times M^{*}$ and for a $\sigma \in \operatorname{Sp}\left(L \times M^{*}\right)$, we set

$$
\chi^{\sigma}(\lambda)=\chi\left(\lambda \sigma^{-1}\right) F_{\sigma-1}(\lambda) \quad\left(\forall \lambda \in L \times M^{*}\right) .
$$

Then $\chi^{\sigma}$ is again a character of $L \times M^{*}$ and $\chi^{\sigma \tau}=\left(\chi^{\sigma}\right)^{\tau}$.

Proposition 1.1. (Generalized Poisson summation formula).

(i ) Let $r(\sigma)\left(\sigma \in \operatorname{Sp}\left(L \times M^{*}\right)\right)$ be a unitary operator in $L^{2}(V)$ which satisfies (1.2). There exist constants $C_{\sigma}^{x}(\mu, \nu)(\mu, \nu \in M / L)$ which satisfy $\theta(r(\sigma) f, \chi(\mu))=\sum_{\nu \in M / L} C_{\sigma}^{\chi}(\mu, \nu) \theta\left(f, \chi^{\sigma}(\nu)\right)(\forall f \in \mathscr{S}(V))$.

(ii) Denote by $C_{\sigma}^{x}$ the matrix of size $[M ; L]$ whose $(\mu, \nu)$-entry $(\mu, \nu \in M / L)$ is $C_{\sigma}^{x}(\mu, \nu)$. Then $C_{\sigma}^{x}$ is a unitary matrix and $C_{o \tau}^{x}=c(\sigma, \tau)$. $C_{\sigma}^{x} C_{\tau}^{x^{\sigma}}$, where $c(\sigma, \tau)$ is a complex number of modulus 1 given in (1.3). 
(iii) Set $\sigma=\left(\begin{array}{ll}a & b \\ c & d\end{array}\right)$ and assume $c$ is non-singular and $r(\sigma)$ is normalized by the formula (1.4). Then the constant $C_{\sigma}^{x}(\mu, \nu)$ is given by

$$
\begin{aligned}
& \operatorname{vol}\left(V^{*} / M^{*}\right)|c|^{1 / 2} C_{o}^{\chi}(\mu, \nu) \\
& =\sum_{l \in L / M^{*} c^{*}} e\left[\frac{1}{2}\left(l+\mu^{\prime},\left(l+\mu^{\prime}\right) a c^{-1}\right)-\left(l+\mu^{\prime}, \nu^{\prime} c^{-1}\right)\right. \\
& \left.\quad+\frac{1}{2}\left(\nu^{\prime}, \nu^{\prime} c^{-1} d\right)+\left(l, v_{x}^{*}\right)\right]
\end{aligned}
$$

(where $\mu^{\prime}=\mu+v_{x}$ and $\nu^{\prime}=\nu+v_{x^{\sigma}}$ ).

Proof. (i) Denote by $M_{\sigma}\left(\sigma \in \operatorname{Sp}\left(L \times M^{*}\right)\right)$ an isometric linear mapping from $\Theta_{\chi}$ onto $\Theta_{x^{\sigma}}$ given by $\left(M_{\sigma} \theta\right)(z)=\theta\left(z \sigma^{-1}\right) F_{\sigma-1}(z)\left(z \in V \times V^{*}\right)$. By (1.7), it is easy to see that

$$
M_{\sigma} T_{\chi}(w)=T_{\chi^{\sigma}}\left(w^{\sigma}\right) M_{\sigma} \quad\left(\forall w \in A(V), \sigma \in \operatorname{Sp}\left(L \times M^{*}\right)\right) .
$$

Hence, it follows from (1.2) and (1.9) that

$$
M_{\sigma} \theta_{\chi(\mu)} r(\sigma) U(w)=T_{\chi^{\sigma}}(w) M_{\sigma} \theta_{\chi(\mu)} r(\sigma) \quad(\forall w \in A(V), \mu \in M / L) .
$$

Thus, $M_{\sigma} \theta_{\chi(\mu)} r(\sigma)$ is a norm preserving linear mapping from $L_{2}(V)$ into $\Theta_{x^{\circ}}$ which commutes with the action of $A(V)$. Therefore, for suitable complex numbers $C_{\sigma}^{x}(\mu, \nu)(\nu \in M / L)$,

$$
\begin{gathered}
\left\{M_{\sigma} \theta_{\chi(\mu)} r(\sigma) f\right\}\left(x, x^{*}\right)=\sum_{\nu \in M / L} C_{\sigma}^{\chi}(\mu, \nu) \theta_{\chi^{\sigma}(\nu)}(f)\left(x, x^{*}\right) \\
\left(\forall f \in \mathscr{S}(V), \forall\left(x, x^{*}\right) \in V \times V^{*}\right) .
\end{gathered}
$$

Evaluating both sides of the above equality for $\left(x, x^{*}\right)=(0,0)$, we obtain the first part of our proposition.

(ii) The linear mappings $M_{\sigma}, r(\sigma), \theta_{\chi_{(\mu)}}$ and $\theta_{\chi^{\sigma(\nu)}}$ are all norm preserving. Furthermore, $\theta_{\chi\left(\mu_{1}\right)} f$ (resp. $\theta_{\chi} \sigma_{\left(\nu_{1}\right)} f$ ) and $\theta_{\chi\left(\mu_{2}\right)} f\left(\right.$ resp. $\theta_{\chi} \sigma_{\left(\nu_{2}\right)} f$ ) are mutually orthogonal if $\mu_{1} \neq \mu_{2}$ (resp. $\nu_{1} \neq \nu_{2}$ ). Hence, the matrix $C_{o}^{x}=$ $\left(c_{\sigma}^{x}(\mu, \nu)\right)$ is unitary. The equality (1.12), together with relations $r(\sigma \tau)=$ $c(\sigma, \tau) r(\sigma) r(\tau)$ and $M_{\sigma \tau}=M_{\tau} M_{\sigma}$, implies that $C_{\sigma \tau}^{\chi}=c(\sigma, \tau) C_{\sigma}^{\chi} C_{\tau}^{x^{\sigma}}$.

(iii) Since $\sigma$ is symplectic, $\sigma^{-1}=\left(\begin{array}{cc}d^{*} & -b^{*} \\ -c^{*} & a^{*}\end{array}\right)$, where $a^{*}, b^{*}$ etc. are adjoint linear mappings of $a, b$ etc.

It follows easily from Lemma 11 of Chap. 1 of Igusa [5], that $r(\sigma) r\left(\sigma^{-1}\right)$ $=1$ if $c$ is non-singular. Put $\nu^{\prime \prime}=\nu+v_{x^{\sigma-1}}$. Then, by (1.10), we have 


$$
\begin{aligned}
\operatorname{vol}\left(V^{*} / M^{*}\right) C_{\sigma-1}^{x}(\mu, \nu)\{r(\sigma) f\}\left(x+\nu^{\prime \prime}\right) \\
=\int_{V^{* / M^{*}}} F_{\sigma}\left(x, x^{*}\right) \sum_{l \in L} e\left[\left(l+\mu^{\prime}, x b+x^{*} d\right)+\left(l, v_{x}^{*}\right)-\left(\nu^{\prime \prime}, x^{*}\right)\right] \\
\quad \times f\left(x a+x^{*} c+l+\mu^{\prime}\right) d x^{*}
\end{aligned}
$$

If $c$ is non-singular, it follows from (1.1),

$$
\begin{aligned}
& F_{\sigma}\left(x, x^{*}\right) e\left[\left(l+\mu^{\prime}, x b+x^{*} d\right)-\left(\nu^{\prime \prime}, x^{*}\right)\right] \\
& \quad=F_{\sigma}\left(x+\nu^{\prime \prime}, x^{*}+\left(-\nu^{\prime \prime} a+l+\mu^{\prime}\right) c^{-1}\right) F_{\sigma}^{-1}\left(\nu^{\prime \prime},\left(-\nu^{\prime \prime} a+l+\mu\right) c^{-1}\right) .
\end{aligned}
$$

Hence, the above integral is equal to

$$
\begin{aligned}
\sum_{l \in L / M^{*} c} F_{\sigma}^{-1}\left(\nu^{\prime \prime},\left(-\nu^{\prime \prime} a+l+\mu^{\prime}\right) c^{-1}\right) e\left[l, v_{\chi}^{*}\right] \\
\quad \times \int_{V^{*}} F_{\sigma}\left(x+\nu^{\prime \prime}, x^{*}\right) f\left(\left(x+\nu^{\prime \prime}\right) a+x^{*} c\right) d x^{*}
\end{aligned}
$$

By (1.4), we see

$$
\begin{aligned}
C_{o-1}^{\chi}(\mu, \nu)= & \operatorname{vol}\left(V^{*} / M^{*}\right)^{-1}|c|^{-1 / 2} \sum_{l \in L / M^{*} c} F_{\sigma}^{-1}\left(\nu^{\prime \prime},\left(-\nu^{\prime \prime} a+l+\mu^{\prime}\right) c^{-1}\right) e\left[l, v_{x}^{*}\right] \\
= & \operatorname{vol}\left(V^{*} / M^{*}\right)^{-1}|c|^{-1 / 2} \sum_{l \in L / M^{*} c} e\left[-\frac{1}{2}\left(l+\mu^{\prime},\left(l+\mu^{\prime}\right) d^{*} c^{*-1}\right)\right. \\
& \left.+\left(l+\mu^{\prime}, \nu^{\prime \prime} c^{*-1}\right)-\frac{1}{2}\left(\nu^{\prime \prime}, \nu^{\prime \prime} c^{*-1} a^{*}\right)\right] e\left[\left(l, v_{x}^{*}\right)\right] .
\end{aligned}
$$

Remark 1.1. Identify $V$ with $\boldsymbol{R}^{n}$ and set $f(x)=\exp \pi \sqrt{-1} x \zeta^{t} x$, where $\zeta$ is a complex symmetric matrix with positive definite imaginary part. Then the equality (1.12) is a classical transformation formula for theta functions.

4. In the following, we set $V=\boldsymbol{R}^{n}$. Take a non-degenerate symmetric $n \times n$ matrix $Q$ and identify $V$ with its dual by setting $(x, y)=$ ${ }^{t} y Q x$. We put $d x=d x_{1} \cdots d x_{n}$. Then the dual measure $d x^{*}$ is given by $d x^{*}=|\operatorname{det} Q| d x$. We denote by $r(\cdot, Q)$ the Weil representation of $\operatorname{Sp}\left(V \times V^{*}\right)$ on $L^{2}(V)$, to emphasize its dependence on $Q$. Identify the group $S L(2, R)$ with a subgroup of $\operatorname{Sp}\left(V \times V^{*}\right)$ by setting

$$
(x, y) \sigma=(x a+y c, x b+y d)\left(x, y \in V, \sigma=\left(\begin{array}{ll}
a & b \\
c & d
\end{array}\right) \in S L(2, \boldsymbol{R})\right) .
$$

By (1.4), we have the following expression for $r(\sigma, Q)\left(\sigma=\left(\begin{array}{ll}a & b \\ c & d\end{array}\right) \epsilon\right.$ $S L(2, R))$ 


$$
r(\sigma, Q) f(x)= \begin{cases}|c|^{-n / 2} \sqrt{|\operatorname{det} Q|} \int_{V} e\left[\frac{a(x, x)-2(x, y)+d(y, y)}{2 c}\right] f(y) d y \\ |a|^{n / 2} e\left[\frac{a b}{2}(x, x)\right] f(a x) & (c=0) .\end{cases}
$$

The group $G L(n, R)$ acts on $L^{2}(V)$, as a group of unitary operators if one puts

$$
(T f)(x)=\sqrt{|\operatorname{det} T|^{-1}} f\left(T^{-1} x\right) .
$$

It is easy to see that

$$
r\left(\sigma,{ }^{t} T^{-1} Q T^{-1}\right) \cdot T=\operatorname{Tr}(\sigma, Q) \quad\left(\forall_{\sigma} \in S L(2, R), T \in G L(n, R)\right) .
$$

We are going to determine the constant $c(\sigma, \tau)$ introduced in (1.3) for $\sigma, \tau \in S L(2, R)$.

Denote by $\mathscr{S}$ the complex upper half plane $\mathscr{S}=\{z \in C ; \operatorname{Im} z>0\}$. For a $\sigma=\left(\begin{array}{ll}a & b \\ c & d\end{array}\right) \in S L(2, R)$ and $z \in \mathfrak{S}_{\mathcal{C}}$, we write

$$
J(\sigma, z)=c z+d \text { and } \sigma \cdot z=(a z+b)(c z+d)^{-1} .
$$

We define a function $\varepsilon(\sigma)$ on $S L(2, R)$ as follows:

$$
\varepsilon(\sigma)= \begin{cases}\sqrt{i} & c>0, \\ i^{(1-\operatorname{sgn} d) / 2} & c=0, \\ \sqrt{i}^{-1} & c<0 .\end{cases}
$$

Take a positive definite symmetric matrix $R$ which satisfies

$$
R Q^{-1} R=Q \text {. }
$$

For $z=u+i v \in \mathfrak{S}$, set

$$
Q_{z}=u Q+i v R .
$$

Let $P_{\nu}(x)$ be a homogeneous polynomial of degree $\nu$ which has the following expression:

$$
P_{\nu}(x)= \begin{cases}1 & \text { for } \nu=0 \\ (r, x),\left(r \in C^{n}, Q r=R r\right) ; & \text { for } \nu=1 \\ \sum c_{r}(r, x)^{\nu},\left(c_{r} \in C, r \in C^{n}, Q r=R r,(r, r)=0\right), & \text { for } \nu \geq 2,\end{cases}
$$

(if $\operatorname{rank}(Q-R)=1$, we assume $\nu \leq 1$ ). 
LEMMA 1.2. Assume $Q$ has $p$ positive and $q$ negative eigenvalues $(p+q=n, p>0)$. Set

$$
F_{z}(x)=e\left[\frac{1}{2} Q_{z}(x)\right] P_{\nu}(x) .
$$

Then

$$
\begin{aligned}
& r(\sigma, Q) F_{z}(x) \\
& \quad=\varepsilon(\sigma)^{p-q} \sqrt{J(\sigma, z)}^{q-p}|J(\sigma, z)|^{-q} J(\sigma, z)^{-\nu} F_{\sigma \cdot z}(x)
\end{aligned}
$$

for every $\sigma \in S L(2, R)$.

Proof. There exists a $T \in G L(n, R)$ which satisfies ${ }^{t} T Q T=\left(\begin{array}{ll}1_{p} & \\ & -1_{q}\end{array}\right)$ and ${ }^{t} T R T=1_{n}$. By (1.14), it is sufficient to prove the lemma under the additional assumption that

$$
Q=\left(\begin{array}{cc}
1_{p} & \\
& -1_{q}
\end{array}\right), \quad R=1_{n}
$$

Set $\sigma=\left(\begin{array}{ll}a & b \\ c & d\end{array}\right)$. If $c=0$, the lemma is immediate. If $c \neq 0$, we have, by a straightforward computation,

$$
\begin{aligned}
& r(\sigma, Q) F_{z}(x) \\
& \quad=|c|^{-n / 2} \sqrt{v-i u-i d / c}^{-p}{\sqrt{v+i u+i d / c^{-q}} J(\sigma, z)^{-\nu} F_{\sigma \cdot z}(x) .}
\end{aligned}
$$

Now lemma follows from a simple observation.

By Lemma 1.2, we have

$$
\left\{\begin{array}{l}
c(\sigma, \tau)=\{\varepsilon(\sigma \tau) / \varepsilon(\sigma) \varepsilon(\tau)\}^{p-q} c_{0}(\sigma, \tau)^{q-p}, \quad \text { where we put } \\
c_{0}(\sigma, \tau)=\sqrt{J(\sigma \tau, i)} / \sqrt{J(\sigma, \tau i)} \sqrt{J(\tau, i)} .
\end{array}\right.
$$

For a $\sigma \in S L(2, R)$, set

$$
r_{0}(\sigma, Q)=\varepsilon(\sigma)^{q-p} r(\sigma, Q)
$$

Let $\mathbb{S}_{1}$ be a Lie group with the underlying manifold $S L(2, R) \times T$ and with the law of composition given by

$$
(\sigma, t)\left(\sigma^{\prime}, t^{\prime}\right)=\left(\sigma \sigma^{\prime}, t t^{\prime} c_{0}\left(\sigma, \sigma^{\prime}\right)\right)
$$

Then a subgroup $\{(\sigma, \pm 1) ; \sigma \in S L(2, R)\}$ of $\mathscr{S}_{1}$ is isomorphic to the twofold covering group of $S L(2, R)$ (see p. 444 of [10]). For a $\tilde{\sigma}=(\sigma, t) \in \mathbb{S}_{1}$, set $r_{0}(\tilde{\sigma}, Q)=t^{p-q} r_{0}(\sigma, Q)$. 
The following lemma is now immediate to see.

LEMMA 1.3. (i) The mapping: $\tilde{\sigma} \mapsto r_{0}(\tilde{\sigma}, Q)$ gives a unitary representation of $\mathscr{S}_{1}$ on $L^{2}(V)$. The space $\mathscr{S}(V)$ is a dense invariant subspace.

(ii) For any $f \in \mathscr{S}(V)$, the mapping $\tilde{\sigma} \mapsto r_{0}(\tilde{\sigma}, Q) f$ is a smooth mapping from $\mathscr{B S}_{1}$ into $\mathscr{S}\left(\boldsymbol{R}^{n}\right)$.

5. The mapping $\sigma \mapsto(\sigma, 1)$ gives a locally isomorphic imbedding of $S L(2, R)$ into $\oiint_{1}$. So, for any element $u$ of the universal enveloping algebra of the Lie algebra of $S L(2, R), r_{0}(u, Q)$ has an obvious meaning as a differential operator on $V$. In particular set

$$
\begin{array}{ll}
C_{Q}=r_{0}(C, Q), & \text { where } C=2 X Y+2 Y X+H^{2}, \\
X=\left(\begin{array}{ll}
0 & 1 \\
0 & 0
\end{array}\right), \quad Y=\left(\begin{array}{ll}
0 & 0 \\
1 & 0
\end{array}\right) \text { and } H=\left(\begin{array}{ll}
1 & \\
& -1
\end{array}\right) .
\end{array}
$$

Then $C_{Q}$ commutes with $r_{0}(\tilde{\sigma}, Q)$ for any $\tilde{\sigma} \in \mathbb{S}_{1}$.

For $\theta \in \boldsymbol{R}$, we write $k_{\theta}=\left(\begin{array}{cc}\cos \theta & \sin \theta \\ -\sin \theta & \cos \theta\end{array}\right)$.

Set $\Re=\left\{\left(k_{\theta}, \varepsilon\right) ; \theta \in R, \varepsilon= \pm 1\right\}$. Then $\Re$ is a compact abelian subgroup of $\mathbb{S}_{1}$. For an integer $m$, we put

$$
\chi_{m}\left(\left(k_{\theta}, \varepsilon\right)\right)=\left(\sqrt{e^{-i \theta}}\right)^{-m} \varepsilon^{m} .
$$

Then $\chi_{m}$ is a character of $\AA$. Take an $f \in \mathscr{S}(V)$ which satisfies

$$
r_{0}(\kappa, Q) f=\chi_{m}(\kappa) f \quad\left(\forall_{\kappa} \in \Re\right) .
$$

If it does not vanish identically, $m$ has the same parity as $p-q .^{(1)}$ For the proof of the next lemma, see $\S 4$ of chap. 1 of [2].

LEMMA 1.4. For $a z=u+i v \in \mathfrak{F}$, set

$$
\sigma_{z}=\left(\begin{array}{cc}
\sqrt{v} & \sqrt{v}^{-1} u \\
& \sqrt{v}^{-1}
\end{array}\right) .
$$

Then

$$
r_{0}\left(\sigma_{z}, Q\right) C_{Q} f=\left\{4 v^{2}\left(\frac{\partial^{2}}{\partial u^{2}}+\frac{\partial^{2}}{\partial v^{2}}\right)-2 i m v \frac{\partial}{\partial u}\right\} r_{0}\left(\sigma_{z}, Q\right) f
$$

Denote by $G$ the connected component of the identity element of the group $\boldsymbol{O}(Q)$ of real linear transformations which leave the quadratic form

(1) For the definition of $p$ and $q$, see Lemma 1.2. 
$Q$ invariant. Then (1.13) gives a unitary representation of $G$ on $L^{2}(V)$ which commutes with $r(\tilde{\sigma}, Q)$ for any $\tilde{\sigma} \in \mathbb{S}_{1}$. Take a $T \in G L(n, R)$ which satisfies ${ }^{t} T Q T=\left(\begin{array}{cc}1_{p} & \\ & -1_{q}\end{array}\right)$ and set

$$
\begin{array}{ll}
X_{i j}=T\left(e_{i j}-e_{j i}\right) T^{-1} & (1 \leq i<j \leqslant p \text { or } p<i<j \leqslant n), \\
Y_{k l}=T\left(e_{k l}+e_{l k}\right) T^{-1} & (1 \leqslant k \leqslant p<l \leqslant n)^{(2)} .
\end{array}
$$

Then $X_{i j}$ and $Y_{k \ell}$ form a base of the Lie algebra of $G$. Set

$$
L_{Q}=\sum_{1 \leq i<j \leq p \text { or } p<i<j \leq n} X_{i j}^{2}+\sum_{1 \leq k \leq p<l \leq n} Y_{k l}^{2} .
$$

Then $L_{Q}$ is the Casimir operator on $G$. The representation (1.13) of $G$ maps $L_{Q}$ to a second order differential operator on $\boldsymbol{R}^{n}$ which we denote by the same symbol $L_{Q}$.

LeMma 1.5. For any $F \in \mathscr{S}(V)$,

$$
C_{Q} F=\left(L_{Q}+n(n-4) / 4\right) F .
$$

Proof. By (1.14), we may assume that $Q=\left(\begin{array}{ll}1_{p} & \\ & -1_{q}\end{array}\right)$. In this case, it is a simple calculation to show that

$$
\begin{aligned}
r(H, Q) F & =\sum_{i=1}^{n} x_{i} \frac{\partial F}{\partial x_{i}}+\frac{n}{2} F,^{(3)} \\
r(X, Q) F & =\pi \sqrt{-1}(x, x) F
\end{aligned}
$$

and

$$
r(Y, Q) F=\sqrt{-1}(4 \pi)^{-1}\left(\sum_{i=1}^{p} \frac{\partial^{2}}{\partial x_{i}^{2}}-\sum_{j=p+1}^{n} \frac{\partial^{2}}{\partial x_{j}^{2}}\right) F
$$

Thus

$$
\begin{aligned}
C_{Q} F= & -(x, x)\left(\sum_{i=1}^{p} \frac{\partial}{\partial x_{i}^{2}}-\sum_{j=p+1}^{n} \frac{\partial^{2}}{\partial x_{j}^{2}}\right) F+\sum_{i=1}^{n} x_{i}^{2} \frac{\partial^{2} F}{\partial x_{i}^{2}} \\
& +(n-1) \sum_{i=1}^{n} x_{i} \frac{\partial F}{\partial x_{i}}+\left(\frac{n^{2}}{4}-n\right) F+2 \sum_{1 \leq i<j \leq n} x_{i} x_{j} \frac{\partial^{2} F}{\partial x_{i} \partial x_{j}} .
\end{aligned}
$$

On the other hand,

(2) We denote by $e_{i j}$ the $n \times n$ matrix whose $(k l)$-entry is $\delta_{k i} \delta_{l j}$.

(3) For the definition of $H, X$ and of $Y$, see (1.18). 


$$
\begin{aligned}
L_{Q} F= & -\sum\left(x_{j} \frac{\partial}{\partial x_{i}}-x_{i} \frac{\partial}{\partial x_{j}}\right)^{2} F+\sum\left(x_{l} \frac{\partial}{\partial x_{k}}+x_{k} \frac{\partial}{\partial x_{l}}\right)^{2} F \\
= & -(x, x)\left(\sum_{i=1}^{p} \frac{\partial^{2}}{\partial x_{i}^{2}}-\sum_{j=p+1}^{n} \frac{\partial^{2}}{\partial x_{i}^{2}}\right) F+2 \sum_{1 \leq i<j \leq n} x_{i} x_{j} \frac{\partial^{2} F}{\partial x_{i} \partial x_{j}} \\
& +(n-1) \sum_{i=1}^{n} x_{i} \frac{\partial}{\partial x_{i}}+\sum_{i=1}^{n} x_{i}^{2} \frac{\partial^{2} F}{\partial x_{i}^{2}} .
\end{aligned}
$$

Hence, $C_{Q}=L_{Q}+n(n-4) / 4$.

6. In the remaining part of this section, we assume $Q$ to be a rational symmetric matrix with $p>0$ positive and $q=n-p$ negative eigenvalues. Take a lattice $L$ in $V$ and denote by $L^{*}$ the lattice dual to $L$ in $V: L^{*}=\left\{x \in V ;(x, y)={ }^{t} x Q y \in Z^{n} ;{ }^{\forall} y \in L\right\}$. We always assume that $L^{*} \supset L$. We denote by $v(L)$ the volume of the fundamental parallelotop of $L$ in $V$ :

$$
v(L)=\int_{R^{n / L}} d x
$$

For an $f \in \mathscr{S}(V)$ and $h \in L^{*} / L$, set $\theta(f, h)=\sum_{l \in L} f(l+h)$.

Proposition 1.6. Let $\sigma=\left(\begin{array}{ll}a & b \\ c & d\end{array}\right) \in S L(2, Z)$ satisfy the following condition (1.21).

$$
a b(x, x) \equiv c d(y, y) \equiv 0 \quad(\bmod 2)\left({ }^{\forall} x, y \in L\right)
$$

Then the following assertions (i) and (ii) hold.

(i) $\theta(r(\sigma, Q) f, h)=\sum_{k \in L^{*} / L} c(h, k)_{\sigma} \theta(f, k)\left({ }^{\forall} f \in \mathscr{S}(V)\right)$, where $c(h, k)_{\sigma}$

$$
=\left\{\begin{array}{l}
\delta_{h, a k} e\left[\frac{a b}{2}(h, h)\right] \quad(c=0) \\
\sqrt{|\operatorname{det} Q|^{-1} v(L)^{-1}|c|^{-n / 2}} \sum_{r \in L / c L} e\left[\frac{a(h+r, h+r)-2(k, h+r)+d(k, k)}{2 c}\right] \\
(c \neq 0)
\end{array}\right.
$$

(ii) Further assume that $c$ is even, $c L^{*} \subset L, c d \neq 0$ and $c(x, x) \equiv 0$ (mod.2) for every $x \in L^{*}$. Let $\left\{\lambda_{1}, \cdots, \lambda_{n}\right\}$ be a $Z$-base of $L$ and set $D=\operatorname{det}\left(\left(\lambda_{i}, \lambda_{j}\right)\right)$. Then 


$$
\begin{aligned}
& \sqrt{i}^{-(p-q) \operatorname{sgn}(c d)} c(h, k)_{o} \\
& = \begin{cases}\delta_{h, d k} e\left[\frac{a b}{2}(h, h)\right] \varepsilon_{d}^{-n}(\operatorname{sgn} c i)^{n}\left(\frac{2 c}{d}\right)^{n}\left(\frac{D}{-d}\right) & (d<0), \\
\delta_{h, d k} e\left[\frac{a b}{2}(h, h)\right] \varepsilon_{d}^{n}\left(\frac{-2 c}{d}\right)^{n}\left(\frac{D}{d}\right) & (d>0),\end{cases}
\end{aligned}
$$

where $\varepsilon_{d}=1$ or $i$ according as $d \equiv 1$ or $d \equiv 3$ (mod. 4$)$.

Proof. (i) We note that the group $S L(2, Z)$ is mapped into a subgroup of $\operatorname{Sp}(L \times L)$ by our embedding of $S L(2, R)$ into $\operatorname{Sp}\left(V \times V^{*}\right)$. Thus, the first half of our proposition is an immediate consequence of Proposition 1.1.

(ii) Let $e_{0}$ be the index of $L$ in $L^{*}$. Denote by $C_{o}$ the matrix of size $e_{0}$ whose $(h, k)$ entry is $c(h, k)_{\sigma}\left(h, k \in L^{*} / L\right)$. If $\sigma, \sigma^{\prime}$ and $\sigma \sigma^{\prime}$ all satisfy the condition (1.21), it follows from the second half of Proposition 1.1 that

$$
C_{\sigma \sigma^{\prime}}=c\left(\sigma, \sigma^{\prime}\right) C_{\sigma} C_{\sigma^{\prime}}
$$

Now set $\sigma^{\prime}=\left(\begin{array}{ll}-b & a \\ -d & c\end{array}\right)$ and $\omega=\left(\begin{array}{ll}1 & -1\end{array}\right)$. Then $\sigma^{\prime}, \omega$ and $\sigma=\sigma^{\prime} \omega$ all satisfy the condition (1.21). By (1.17), we have

$$
c\left(\sigma^{\prime}, \omega\right)=\sqrt{i}^{(p-q) \operatorname{sgn}(c d)} .
$$

Hence

$$
\begin{aligned}
c(h, k)_{o} & =\sqrt{i}^{(p-q) \operatorname{sgn}(c d)}|\operatorname{det} Q|^{-1} v(L)^{-2}|d|^{-n / 2} \\
& \times \sum_{r \in L / d L} \sum_{l \in L^{*} / L} e\left[\frac{-b(h+r, h+r)-2(l, h+r)+c(l, l)}{-2 d}\right] e[-(l, k)] .
\end{aligned}
$$

Since $c L^{*} \subset L$, the mapping $l \mapsto d l$ induces an automorphism of $L^{*} / L$. Taking into account the assumption that $c(x, x) \in 2 Z\left({ }^{\forall} x \in L^{*}\right)$, we have,

$$
\begin{array}{rl}
\sum_{l \in L^{*} / L} e[ & -b(h+r, h+r)-2(l, h+r)+c(l, l) \\
-2 d & e[-(l, k)] \\
& =e\left[\frac{b(h+r, h+r)}{2 d}\right] \sum_{l \in L^{*} / L} e[(l, h-d k)]=e_{0} e\left[\frac{b(h+r, h+r)}{2 d}\right] \delta_{h, d k}
\end{array}
$$

On tne other hand, the Poisson summation formula implies that $|\operatorname{det} Q|^{-1} v(L)^{-2} e_{0}=1$. Furthermore, 


$$
\begin{aligned}
\sum_{r \in L / d L} e\left[\frac{b(h+r, h+r)}{2 d}\right] & =\sum_{r \in L / d L} e\left[\frac{b(a d h+r, a d h+r)}{2 d}\right] \\
& =e\left[\frac{a b(h, h)}{2}\right] \sum_{r \in L / d L} e\left[\frac{b(r, r)}{2 d}\right]
\end{aligned}
$$

Thus, we have,

$$
\begin{aligned}
C_{o}(h, k)= & \delta_{h, d k} \sqrt{i}^{(p-q) \operatorname{sgn}(c d)} e\left[\frac{a b}{2}(h, h)\right] \\
& \times|d|^{-n / 2} \sum_{r \in L / d L} e\left[\frac{b(r, r)}{2 d}\right] .
\end{aligned}
$$

Now the argument used in the proof of Proposition 2.1 of [10] is applicable with a slight modification to show that

$$
|d|^{-n / 2} \sum_{r \in L / d L} e\left[\frac{b(r, r)}{2 d}\right]= \begin{cases}\varepsilon_{d}^{-n}(\operatorname{sgn}(c) i)^{n}\left(\frac{2 c}{d}\right)^{n}\left(\frac{D}{-d}\right) & (d<0), \\ \varepsilon_{d}^{n}\left(\frac{-2 c}{d}\right)^{n}\left(\frac{D}{d}\right) & (d>0) .\end{cases}
$$

Remark 1.2. Proposition 1.1 appears in many literatures. Here, we refer only to Hilfssatz 1 of [12] and Proposition 2.1 of [10].

7. The group $G$ is, as in $\mathbf{5}$, the connected component of the identity of the real orthogonal group of $Q$. Let $\Gamma$ be the subgroup of $G$ formed by all elements which leave the lattice $L$ invariant and leave $L^{*} / L$ pointwise fixed. Then, as a function on $G, \theta(g \cdot f, h)^{(4)}\left(f \in \mathscr{S}(V), g \in G, h \in L^{*} / L\right)$ is left $\Gamma$-invariant and slowly increasing on $\Gamma \backslash G^{(5)}$. Take a rapidly decreasing function $\Phi$ on $\Gamma \backslash G$ and put

$$
\theta(f, \Phi ; h)=\int_{\Gamma \backslash G} \theta(g \cdot f, h) \Phi(g) d g,
$$

where $d g$ is a Haar measure on $G$. Now assume that $f$ satisfies (1.19) and set

$$
\Theta(z, f, \Phi ; h)=v^{-m / 4} \theta\left(r\left(\sigma_{z}, Q\right) f, \Phi ; h\right)
$$

for $z=u+i v \in \mathfrak{S} \cdot{ }^{(6)}$

(4) For the definition of $g \cdot f$, see (1.13).

(5) For the definitions of slowly increasing functions and rapidly decreasing functions on $\Gamma \backslash G$ see [3].

(6) The notation $\sigma_{z}$ is introduced in Lemma 1.4 . 
If no confusion is likely, we write simply

$$
\Theta(z, h)=\Theta(z, f, \Phi ; h) .
$$

Prosition 1.7. Assume $f$ satisfies (1.19).

(i) If $\gamma=\left(\begin{array}{ll}a & b \\ c & d\end{array}\right) \in S L(2, Z)$ satisfies the condition (1.21), then

$$
\sqrt{i}^{(p-q) \operatorname{sgn} c} \sqrt{J(\gamma, z)}{ }^{-m} \Theta(\gamma \cdot z, h)=\sum_{k \in L^{*} / L} c(h, k)_{\gamma} \Theta(z, k) \quad(c \neq 0) .
$$

(ii) Assume that $\Phi$ satisfies the differential equation $L_{Q} \Phi=\lambda \Phi$ on G. ${ }^{(7)}$ Then

$$
\begin{aligned}
\left\{4 v ^ { 2 } \left(\frac{\partial^{2}}{\partial u^{2}}\right.\right. & \left.\left.+\frac{\partial^{2}}{\partial v^{2}}\right)-2 i m v\left(\frac{\partial}{\partial u}+i \frac{\partial}{\partial v}\right)\right\} \Theta(z, h) \\
& =\left\{\lambda-m\left(\frac{m}{4}-1\right)+n\left(\frac{n}{4}-1\right)\right\} \Theta(z, h) .
\end{aligned}
$$

Proof. (i) It follows easily from (1.17) that

$$
r(\gamma, Q) r\left(\sigma_{z}, Q\right)=r\left(\sigma_{(\gamma \cdot z)}, Q\right) r\left(k_{\theta}, Q\right),
$$

where $e^{-i \theta}=J(\gamma, z) /|J(\gamma, z)|$ and $k_{\theta}=\left(\begin{array}{cc}\cos \theta & \sin \theta \\ -\sin \theta & \cos \theta\end{array}\right)$. Since $f$ satisfies (1.19),

$$
r\left(k_{\theta}, Q\right) f=\sqrt{i}^{(p-q) \mathrm{sgn} c} \sqrt{J(\gamma, z) /|J(\gamma, z)|^{-m}} f
$$

(see $\left.\left(1.17^{\prime}\right)\right)$. So, by Proposition 1.6, we have

$$
\sqrt{i}^{(p-q) \operatorname{sgn} c} \sqrt{J(\gamma, z)}{ }^{-m} \Theta(\gamma \cdot z, h)=\sum_{k \in L^{*} / L} c(h, k)_{r} \Theta(z, k) .
$$

(ii) By Lemma 1.4, we have

$$
\begin{gathered}
\left\{4 v^{2}\left(\frac{\partial^{2}}{\partial u^{2}}+\frac{\partial^{2}}{\partial v^{2}}\right)-2 i m v\left(\frac{\partial}{\partial u}+i \frac{\partial}{\partial v}\right)\right\} \Theta(z, f, \Phi ; h) \\
=m\left(1-\frac{m}{4}\right) \Theta(z, h)+\Theta\left(z, C_{Q} f, \Phi ; h\right) .
\end{gathered}
$$

Applying Lemma 1.3, Lemma 1.5 and integration by part, we have (1.23).

Remark 1.3. For Proposition 1.7, we refer to [7], [8], [9] and [12].

(7) Differential operator $L_{Q}$ is given by (1.20). 


\section{$\S 2$. Construction of modular forms of half integral weight from given forms of even integral weight.}

1. Let $Q$ be a $3 \times 3$ rational symmetric matrix which has 2 positive and one negative eigen-values. We assume that the rational quadratic form associated with $Q$ is a zero form. In other words, the equation $(x, x)={ }^{t} x Q x=0$ has a non-zero solution in $Q^{3}$. Then there exists a $T \in G L(3, Q)$ which satisfies $(T x, T x)={ }^{t} x^{t} T Q T x=M\left(x_{2}^{2}-4 x_{1} x_{3}\right)$, where $M$ is a positive rational number.

Without loss of generality, we may put

$$
(x, y)={ }^{t} y Q x=M\left(x_{2} y_{2}-2 x_{1} y_{3}-2 x_{3} y_{1}\right) \quad\left(x, y \in R^{3}\right) .
$$

We identify $\boldsymbol{R}^{3}$ with the vector space of real binary quadratic forms in indeterminates $X$ and $Y$ via the mapping

$$
x \mapsto x(X, Y)=x_{1} X^{2}+x_{2} X Y+x_{3} Y^{2} .
$$

We denote by $d_{x}$ the discriminant of $x(X, Y)$. Namely, $d_{x}=x_{2}^{2}-4 x_{1} x_{3}$. Let $\rho$ be a representation of $S L(2, R)$ on $R^{3}$ given by

$$
\{\rho(g) \cdot x\}(X, Y)=x(a X+c Y, b X+d Y)\left(g=\left(\begin{array}{ll}
a & b \\
c & d
\end{array}\right)\right) .
$$

Then we have

$$
\rho(g) \cdot x=\left(x_{1}, x_{2}, x_{3}\right)\left(\begin{array}{ccc}
a^{2} & 2 a c & c^{2} \\
a b & a d+b c & c d \\
b^{2} & 2 b d & d^{2}
\end{array}\right) .
$$

It is well-known that $\rho$ gives an isomorphic mapping from $S L(2, R) / \pm 1$ onto the connected component of the neutral element of the orthogonal group of $Q$.

2. Let $\Gamma$ be a congruence subgroup of $S L(2, Z)$ and let $\xi$ be a character of $\Gamma$. In this paper we always assume that $-1 \in \Gamma$ and that the kernel of $\xi$ is also a congruence subgroup of $S L(2, Z)$. A holomorphic function $\varphi$ on the upper half-plane $\mathfrak{F}$ is said to be a holomorphic cusp form of weight $2 k$ ( $k$ is a positive integer) and of character $\xi$ with respect to $\Gamma$ if $\varphi$ satisfies the following two conditions (2.3) and (2.4).

$$
\varphi(\gamma \cdot z)=\xi(\gamma) J(\gamma, z)^{2 k} \varphi(z) \quad(\forall \gamma \in \Gamma) \quad \text { (see (1.15)). }
$$




$$
\varphi \text { vanishes at each cusp of } \Gamma \text {. }
$$

We denote by $S_{2 k}(\Gamma, \xi)$ the space of all such cusp forms. For a $\varphi \in S_{2 k}(\Gamma, \xi)$, set

$$
\Phi(g)=J(g, i)^{-2 k} \varphi(g \cdot i) \quad(g \in S L(2, R)) .
$$

Then $\Phi(g)$ is a function on $S L(2, R) / \pm 1$ which satisfies $\Phi\left(\gamma g k_{\theta}\right)=\xi(\gamma) e^{2 k i \theta} \Phi(g)$ for $\gamma \in \Gamma$ and for

$$
k_{\theta}=\left(\begin{array}{cc}
\cos \theta & \sin \theta \\
-\sin \theta & \cos \theta
\end{array}\right) \quad(\theta \in \boldsymbol{R}) .
$$

Since $\rho$ imbeds $S L(2, R) / \pm 1$ into the orthogonal group of $Q$, we can regard $\Phi$ as a function on the connected component of the identity of the orthogonal group of $Q$. We have

$$
L_{Q} \Phi=\frac{1}{4} \rho(C) \Phi=k(k-1) \Phi
$$

(definitions of $L_{Q}$ and $C$ are given in (1.20) and (1.18)). Let $L$ be a lattice in $Q^{3}$ on which the bilinear form $(x, y)$ given by (2.1) is integral valued. Denote by $L^{*}$ the lattice dual to $L$ with respect to this bilinear form. The group $\Gamma$ operates on $\boldsymbol{R}^{3}$ through the representation $\rho$. Assume that $\Gamma$ leaves the lattice $L$ invariant. Then $\Gamma$ induces a permutation on $L^{*} / L$. We further assume that there exists a function $\nu$ on $L^{*} / L$ which satisfies

$$
\nu(\rho(\gamma) \cdot x)=\xi^{-1}(\gamma) \nu(x) \quad\left(\forall x \in L^{*} / L,{ }^{\forall} \in \Gamma\right) .
$$

We fix a Haar measure $d g$ on $S L(2, \boldsymbol{R})$ by setting $d g=d x_{2} d x_{3} d x_{4} /\left|x_{4}\right|$ for $g=\left(\begin{array}{ll}x_{1} & x_{2} \\ x_{3} & x_{4}\end{array}\right)$. We also write $G=S L(2, R)$.

Take an $f \in \mathscr{S}\left(\boldsymbol{R}^{3}\right)$ which satisfies (1.19) for $m=2 k+1$ and put

$$
\begin{aligned}
v^{(2 k+1) / 4} \Theta(z, f, \varphi, L, \nu) \\
\quad=\int_{\Gamma \backslash G} \sum_{x \in L^{*}} \nu(x)\left\{r\left(\sigma_{z}, Q\right) f\right\}\left(\rho\left(g^{-1}\right) \cdot x\right) \Phi(g) d g,
\end{aligned}
$$

where $z=u+i v \in \mathfrak{S}$ and $\sigma_{z}$ is as Lemma 1.4. If no confusion is likely, we write

$$
\Theta(z, f, \varphi, L, \nu)=\Theta(z, \varphi) .
$$

It follows from Proposition 1.7 that 


$$
\left\{4 v^{2}\left(\frac{\partial^{2}}{\partial^{2} u}+\frac{\partial^{2}}{\partial v^{2}}\right)-2 i(2 k+1) v\left(\frac{\partial}{\partial u}+i \frac{\partial}{\partial v}\right)\right\} \Theta(z, \varphi)=0
$$

So, it would be natural to expect that $\Theta(z, \varphi)$ is a holomorphic function of $z$ on $\mathfrak{S}$. In the next paragraph we will compute the coefficients of Fourier expansion of $\Theta(z, \varphi)$ with respect to $u$ and show that it is actually a holomorphic function of $z$.

3. To state our next proposition, we are going to associate an oriented curve $C(x, \Gamma)$ on the upper half-plane to a rational binary quadratic form $x$ with positive discriminant. Take a $g \in S L(2, R)$ which satisfies $\{\rho(g) \cdot x\}(X, Y)=\sqrt{d_{x}} X Y\left(d_{x}=x_{2}^{2}-4 x_{1} x_{3}\right)$. Denote by $\Gamma_{x}$ the isotropy subgroup of $x$ in $\Gamma$. Then if $\Gamma_{x} \neq \pm 1$, it is known that $\Gamma_{x} / \pm 1$ is an infinite cyclic group. If $\Gamma_{x}= \pm 1$, we denote by $C(x, \Gamma)$ the geodesic (with respect to the Poincaré metric) from $\omega_{1}=g^{-1} \cdot \infty$ to $\omega_{2}=g^{-1} \cdot 0$. We note that $\omega_{1}$ and $\omega_{2}$ are two roots of the equation $x(1,-X)=0$ in $X$. If $\Gamma_{x} / \pm 1$ is infinitely cyclic, we take a generator $\gamma_{x}$ of $\Gamma_{x} / \pm 1$ so that $g \gamma_{x} g^{-1}$ is a diagonal matrix whose left upper entry is positive and smaller than 1 . In this case we denote by $C(x, \Gamma)$ any rectifiable curve in $\mathfrak{S}$ from $z$ to $\gamma_{x} \cdot z$ ( $z$ is an arbitrary point in $\mathfrak{S}$ ). Two points of $Q^{3}$ (= the space of rational binary quadratic forms) are said to be $\Gamma$-equivalent if one is transformed into another by a suitable element of $\Gamma$.

Proposition 2.1. The notation being as above, we have

$$
\Theta(z, f, \varphi, L, \nu)=c(f) \sum \nu(x) e[z(x, x) / 2] \int_{c(x, \Gamma)} \varphi(z) x(1,-z)^{k-1} d z,
$$

where the summation is taken over all $\Gamma$-equivalence classes in $L^{*}$ with positive discriminant and $x(1,-z)$ and $c(f)$ is given by the following formula.

$$
\begin{gathered}
x(1,-z)=x_{1}-x_{2} z+x_{3} z^{2} \\
c(f)=2^{-1} \exp (M \pi) \int_{x_{2}^{2}-4 x_{1} x_{3}=1} f(x)\left(x_{1}-i x_{2}-x_{3}\right)^{-k} \frac{d x_{1} d x_{3}}{\left|x_{2}\right|}
\end{gathered}
$$

Proof. The function $v^{(2 k+1) / 4} \Theta(z, f, \varphi, L, \nu)$ is given by the integral in the right hand side of (2.7). Since $\varphi$ is a cusp from, it is easy to see that the integral is absolutely convergent even if each term of the summation in the integrand is replaced by its absolute value. Hence, we can change the order of summation and integration freely. Denote by 
$\{x\}$ the complete set of representatives of $\Gamma$-equivalence classes in $L^{*}$. For each $x, \Gamma_{x}$ (resp. $G_{x}$ ) is the isotropy subgroup of $x$ in $\Gamma$ (resp. $G$ ). We choose invariant measures $d \mu_{x}^{1}$ and $d \mu_{x}^{2}$ on $G_{x}$ and on $G_{x} \backslash G$ respectively which satisfy

$$
\int_{G} F(g) d g=\int_{G_{x} \backslash G} d \mu_{x}^{2}(\dot{g}) \int_{G_{x}} F(h \dot{g}) d \mu_{x}^{1}(h)
$$

for any integrable function $F$ on $G$. Then we have

$$
\begin{aligned}
v^{(2 k+1) / 4} \Theta(z, \varphi)= & \sum_{\{x\}} \nu(x) \int_{\Gamma_{x \backslash G}}\left\{r\left(\sigma_{z}, Q\right) f\right\}\left(\rho\left(g^{-1}\right) \cdot x\right) \Phi(g) d g \\
= & \sum_{\{x\}} \nu(x) \int_{G_{x} \backslash G}\left\{r\left(\sigma_{z}, Q\right) f\right\}\left(\rho\left(\dot{g}^{-1}\right) \cdot x\right) \\
& \times\left(\int_{\Gamma_{x} \backslash G_{x}} \Phi(h \dot{g}) d \mu_{x}^{1}(h)\right) d \mu_{x}^{2}(\dot{g}) .
\end{aligned}
$$

It follows from (2.6) that $\xi$ is trivial on $\Gamma_{x}$ if $\nu(x) \neq 0$. The proof of Proposition 2.1 has now been reduced to the proof of the following sublemma.

Sublemma. Assume that $\xi$ is trivial on $\Gamma_{x}\left(x \in L^{*}\right)$.

(i) If $(x, x)={ }^{t} x Q x=M d_{x} \leqslant 0$, then

$$
\int_{\Gamma_{x} \backslash G_{x}} \Phi(h g) d \mu_{x}^{1}(h)=0
$$

(ii) If $(x, x)>0$, then

$$
\begin{aligned}
v^{-(2 k+1) / 4} & \int_{\Gamma_{x} \backslash G}\left\{r_{0}\left(\sigma_{z}, Q\right) f\right\}\left(\rho\left(g^{-1}\right) \cdot x\right) \cdot \Phi(g) d g \\
& =c(f) e\left[\frac{z}{2}(x, x)\right] \int_{C(x, \Gamma)} \varphi(z) x(1,-z)^{k-1} d z .
\end{aligned}
$$

Proof of Sublemma. (i) Assume $(x, x)<0$, then $\Gamma_{x}$ is a finite group and $G_{x}$ is conjugate to $S O(2)$ in $G$. Denote by $w$ the order of the finite group $\Gamma_{x}$. Then the left side of (2.9) is equal to $w^{-1} J(g, i)^{-2 k} \Psi(z)$, where we put $z=g \cdot i$ and

$$
\Psi(z)=\int_{G_{x}} J(h, z)^{-2 k} \varphi(h \cdot z) d \mu_{x}^{1}(h)
$$

Now it is easy too see that $\Psi(z)$ is a holomorphic function on $\mathfrak{F}$ which satisfies $\Psi(h z)=J(h, z)^{2 k} \Psi(z)$ for any $h \in G_{x}$. It is well-known that such 
a function vanishes identically (we are assuming that $k$ is a positive integer). Next, assume $(x, x)=0, x \neq 0$. There exists a $\gamma \in S L(2, Z)$ which satisfies $\rho(\gamma) \cdot x=(s, 0,0)(s \in \boldsymbol{Q}-\{0\})$. For any $u \in \boldsymbol{R}$, set $n(u)$ $=\left(\begin{array}{cc}1 & u \\ & 1\end{array}\right) \in G$. Then, we have $\gamma G_{x} \gamma^{-1}=\{ \pm n(u) ; u \in R\}$ and $\gamma \Gamma_{x} \gamma^{-1}=$ $\{ \pm n(m N) ; m \in Z\}$ for a suitable positive number $N>0$. Thus the left side of (2.9) is equal to, up to a positive constant factor,

$$
\begin{aligned}
\int_{0}^{N} J\left(\gamma^{-1} n\right. & (u) \gamma g, i)^{-2 k} \varphi\left(\gamma^{-1} n(u) \gamma g \cdot i\right) d u \\
& =J(\gamma g, i)^{-2 k} \int_{0}^{N} J\left(\gamma^{-1}, z+u\right)^{-2 k} \varphi\left(\gamma^{-1} \cdot(z+u)\right) d u,
\end{aligned}
$$

where we put $z=\gamma g \cdot i$.

We note that $\gamma^{-1} \cdot \infty$ is a cusp of $\Gamma$ and $\Gamma_{x}$ is the subgroup of $\Gamma$ which leaves $\gamma^{-1} \cdot \infty$ fixed. Since $\varphi$ is a cusp form, the above integral is zero. Finally assume $x=0$. In this case $G_{x}=G$ and $\Gamma_{x}=\Gamma$. Let $\mathscr{F}$ be a fundamental domain in the upper half plane $\mathfrak{S}$ with respect to the action of $\Gamma$. Then a fundamental domain in $G$ with respect to the left action of $\Gamma$ is given by

$$
\left\{\left(\begin{array}{cc}
\sqrt{v} & \sqrt{v}^{-1} u \\
& \sqrt{v}^{-1}
\end{array}\right)\left(\begin{array}{cc}
\cos \theta & \sin \theta \\
-\sin \theta & \cos \theta
\end{array}\right) ; z=u+i v \in \mathscr{F}, 0 \leqslant \theta<\pi\right\} .
$$

Thus, the left side of (2.9) is, up to a positive constant factor, equal to

$$
\int_{\mathscr{F}} v^{k-2} \varphi(u+i v) d u d v \int_{0}^{\pi} e^{2 k i \theta} d \theta=0
$$

The proof of the first half of the sublemma is now complete.

(ii) Assume $(x, x)>0$. Set $d_{x}=x_{2}^{2}-4 x_{1} x_{3}\left(=M^{-1}(x, x)\right)$. There exists a $g_{1} \in G$ which satisfies $\rho\left(g_{1}\right) \cdot x=\sqrt{d_{x}}(0,1,0)$. Set $S_{t}=g_{1}^{-1}\left(\begin{array}{ll}t & \\ & t^{-1}\end{array}\right) g_{1}$ $(t>0)$. Then the group $G_{x}$ is given by $\left\{ \pm S_{t} ; t>0\right\}$. If $\Gamma_{x} \neq \pm 1, \Gamma_{x}$ is generated by $\pm S_{t_{0}}$ for a suitable $t_{0}<1$. We may assume that the invariant measure $d \mu_{x}^{1}$ on $G_{x}$ is given by

$$
d \mu_{x}^{1}\left(S_{t}\right)=\frac{d t}{t}
$$

We are going to show that $d\left(S_{t} \cdot w\right)=-2{\sqrt{d_{x}}}^{-1} x\left(1,-S_{t} \cdot w\right) d t / t$ for any 
$w \in \mathfrak{S}$, where $x(X, Y)$ is a binary quadratic form given by $x(X, Y)=$ $x_{1} X^{2}+x_{2} X Y+x_{3} Y^{2}$. First, we note the following equality.

$$
x(1,-z)=J(g, z)^{2}(\rho(g) \cdot x)(1,-g \cdot z) \quad(\forall g \in G, z \in \mathfrak{S}) .
$$

Since $g_{1} \cdot\left(S_{t} \cdot w\right)=t^{2}\left(g_{1} \cdot w\right), J\left(g_{1}, S_{t} \cdot w\right)^{-2} d\left(S_{t} \cdot w\right)=2 t\left(g_{1} \cdot w\right) d t$. Replacing $z$ by $S_{t} \cdot w$ and $g$ by $g_{1}$ in (2.12), we have $x\left(1,-S_{t} \cdot w\right)=J\left(g_{1}, S_{t} \cdot w\right)^{2} \sqrt{d_{x}}$ $\cdot\left(-g_{1} \cdot S_{t} \cdot w\right)=-J\left(g_{1}, S_{t} \cdot w\right)^{2} \sqrt{d_{x}} t^{2}\left(g_{1} \cdot w\right)$. Thus, $d\left(S_{t} \cdot w\right)=-2 \sqrt{d_{x}}{ }^{-1} x(1$, $\left.-S_{t} \cdot w\right) d t / t$.

In (2.12), replace $x$ by $\rho\left(S_{t} \cdot g\right)^{-1} \cdot x, g$ by $S_{t} \cdot g$ and $z$ by $i$, then we have

$$
\left(\rho(g)^{-1} x\right)(1,-i)=J\left(S_{t} g, i\right)^{2} x\left(1,-S_{t} g \cdot i\right) .
$$

Hence,

$$
\begin{aligned}
\int_{\Gamma_{x} \backslash G_{x}} \Phi(h \dot{g}) d \mu_{x}^{1}(h)= & \int_{I} J\left(S_{t} \dot{g}, i\right)^{-2 k} \varphi\left(S_{t} \dot{g} \cdot i\right) \frac{d t}{t} \\
= & -2^{-1} \sqrt{d_{x}}\left\{\rho\left(\dot{g}^{-1}\right) x(1,-i)^{-k}\right. \\
& \int_{I} x\left(1,-S_{t} \cdot w\right)^{k-1} \varphi\left(S_{t} \cdot w\right) d\left(S_{t} \cdot w\right)
\end{aligned}
$$

where $w=g \cdot i$ and $I=(0, \infty)$ or $\left(t_{0}, 1\right)$ according as $\Gamma_{x}=+1$ or not. We note that the integral

$$
\begin{array}{r}
-\int_{I} x\left(1,-S_{t} \cdot w\right)^{k-1} \varphi\left(S_{t} \cdot w\right) d\left(S_{t} \cdot w\right) \\
=\int_{C(x, \Gamma)} x(1,-z)^{k-1} \varphi(z) d z
\end{array}
$$

is independent of $w$.

Set $f_{z}(x)=\left\{r_{0}\left(\sigma_{z}, Q\right) f\right\}(x)$.

To prove (2.10) it remains to show that

$$
\begin{aligned}
& 2^{-1} \sqrt{d_{x}} v^{-(2 k+1) / 4} \int_{G_{x} \backslash G} f_{z}\left(\rho\left(\dot{g}^{-1}\right) \cdot x\right)\left\{\rho(\dot{g})^{-1} \cdot x\right\}(1,-i)^{-k} d \mu_{x}^{2}(\dot{g}) \\
& \quad=c(f) e\left[\frac{z}{2}(x, x)\right]
\end{aligned}
$$

where $d \mu_{x}^{2}$ is the invariant measure on $G_{x} \backslash G$ which satisfies (2.8) for $d \mu_{x}^{1}$ given by (2.11).

As a function of $z=u+i v$, the left side of (2.13) is equal to $e[(x, x) u / 2]$ - $F(v)$ for a suitable smooth function $F$ of $v$, since 


$$
f_{z}(x)=v^{3 / 4} e\left[\frac{u}{2}(x, x)\right] f(\sqrt{v} x)
$$

By a straightforward computation one can see that

$$
L_{Q} \cdot x(1,-i)^{-k}=k(k-1) x(1,-i)^{-k},
$$

where $L_{Q}$ is a differential operator on $R^{3}$ given by (1.20). As $f$ satisfies (1.19), for $m=2 k+1$, it follows from Lemma 1.3 and Lemma 1.4 that

$$
\left\{4 v^{2}\left(\frac{\partial^{2}}{\partial u^{2}}+\frac{\partial^{2}}{\partial v^{2}}\right)-2 i(2 k+1) v\left(\frac{\partial}{\partial u}+i \frac{\partial}{\partial v}\right)\right\} F(v) e\left[\frac{u}{2}(x, x)\right]=0 .
$$

Hence $F(v)$ is a linear combination of $e[v(x, x) i / 2]$ and

$$
e\left[\frac{i}{2} v(x, x)\right] \int_{1}^{v} e[-i t(x, x)] t^{-(2 k+1) / 2} d t
$$

However, the left side of (2.13) is bounded as $v \rightarrow+\infty$. Thus, we obtain $F(v) e[u(x, x) / 2]=c e[z(x, x) / 2]$, where $c$ is a constant independent of $z$. On the other hand, it is easy to see that the left side of (2.13) is equal to

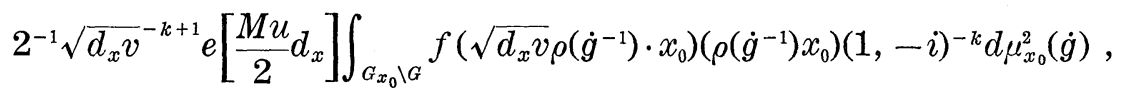

where $x_{0}=(0,1,0)$.

Hence,

$$
\begin{aligned}
c & =2^{-1} \exp \pi M \int_{G_{x_{0} \backslash G}} f\left(\rho\left(\dot{g}^{-1}\right) x_{0}\right)\left\{\rho\left(\dot{g}^{-1}\right) x_{0}\right\}(1,-i)^{-k} d \mu_{x}^{2}(\dot{g}) \\
& =2^{-1} \exp \pi M \int_{x_{2}^{2}-4 x_{1} x_{3}=1} f(x)\left(x_{1}-i x_{2}-x_{3}\right)^{-k} \frac{d x_{1} d x_{3}}{\left|x_{2}\right|}
\end{aligned}
$$

Thus the proof of the second part of Proposition 2.1 is now complete.

Remark 2.1. Set

$$
f(x)=\left(x_{1}-i x_{2}-x_{3}\right)^{k} \exp -\left\{M \pi\left(2 x_{1}^{2}+x_{2}^{2}+2 x_{3}^{2}\right)\right\} .
$$

It follows from Lemma 1.2 that $f$ satisfies (1.19) for $m=2 k+1$. Moreover, it is easy to see that

$$
c(f)=2^{-1} \exp (\pi M) \int_{x_{2}^{2}-4 x_{1} x_{3}=1} \exp \left\{-\pi M\left(2 x_{1}^{2}+x_{2}^{2}+2 x_{3}^{2}\right)\right\} \frac{d x_{1} d x_{3}}{\left|x_{3}\right|}
$$




$$
=\frac{\pi}{2 \sqrt{2 M}} .
$$

Hence, there exists an $f \in \mathscr{S}\left(\boldsymbol{R}^{3}\right)$ which satisfies (1.19) and $c(f) \neq 0$. In the following we write, for $\varphi \in S_{2 k}(\Gamma, \xi)$,

$$
\begin{aligned}
\Theta(z, \varphi, L, \nu) & =c(f)^{-1} \Theta(z, f, \varphi, L, \nu) \\
& =\sum_{x} \nu(x) \int_{C(x, \Gamma)} \varphi(w) x(1,-w)^{k-1} d w e\left[\frac{z}{2}(x, x)\right],
\end{aligned}
$$

where the summation is taken over all $\Gamma$-equivalence classes of $L$ with positive discriminant.

4. For a positive integer $N$, put

$$
\begin{aligned}
\Gamma_{0}(N)=\left\{\left[\begin{array}{ll}
a & b \\
c & d
\end{array}\right] \in S L_{2}(Z) \mid c \equiv 0\right. & (\bmod . N)\} \\
\Gamma(N) & =\left\{\left[\begin{array}{ll}
a & b \\
c & d
\end{array}\right] \in \Gamma_{0}(N) \mid \begin{array}{ll}
b \equiv 0 & (\bmod . N) \\
a \equiv d \equiv 1 & (\bmod N)
\end{array}\right\}
\end{aligned}
$$

Further, put

$$
\begin{gathered}
\theta(z)=\sum_{n=-\infty}^{\infty} e\left[n^{2} z\right], \\
j(\gamma, z)=\theta(\gamma \cdot z) / \theta(z) \quad \text { for } \gamma \in \Gamma_{0}(4) .
\end{gathered}
$$

It is known (see (1.10) of [10]) that

$$
j\left(\left[\begin{array}{ll}
a & b \\
c & d
\end{array}\right], z\right)=\varepsilon_{d}^{-1}\left(\frac{c}{d}\right)(c z+d)^{1 / 2} .
$$

A holomorphic function $\psi$ on the upper half plane is said to be a holomorphic cusp form of weight $(2 k+1) / 2$ and of level $N$ (, where $N$ is a positive integer divisible by 4,) if it satisfies the following conditions $(2.17)_{1}$ and $(2.17)_{2}$.

$$
\psi(\gamma \cdot z)=j(\gamma, z)^{2 k+1} \psi(z) \quad(\forall \gamma \in \Gamma(N)),
$$

At each cusp of $\Gamma(N), \psi(z)$ vanishes.

As in 2 let $\Gamma$ be a congruence subgroup of $S L(2, Z)$ and $\xi$ be a character of $\Gamma$ whose kernel is also a congruence subgroup. Let $L$ be a lattice in $Q^{3}$ and $L^{*}$ be the lattice dual to $L$ with respect to the bilinear form (2.1). We assume that $L^{*} \supset L,-1 \in \Gamma$ and that $L$ is invariant 
under the action of $\Gamma$. Take a function $\nu$ on $L^{*} / L$ which satisfies (2.6). Take a $Z$-base $\left\{\lambda_{1}, \lambda_{2}, \lambda_{3}\right\}$ of $L$ and set $D=\operatorname{det}\left(\left(\lambda_{i}, \lambda_{j}\right)\right)$.

THEOREM 1. Notations being as above, if $\varphi \in S_{2 k}(\Gamma, \xi)$, the function $\Theta(z, \varphi)=\Theta(z, \varphi, L, \nu)$ given by (2.14) is a holomorphic cusp form of weight $(2 k+1) / 2$ and of level $N$, if $N$ is divisible by $8 D$.

Proof. Take an $f \in \mathscr{S}\left(\boldsymbol{R}^{3}\right)$ which satisfies (1.19) for $m=2 k+1$ and $c(f)=1$. Then we have $\Theta(z, \varphi)=\Theta(z, f, \varphi, L, \nu)$ (see 2.7). Denote by $\Gamma_{0}$ the kernel of the character $\xi$ of $\Gamma$. By the assumption, $\Gamma_{0}$ is a congruence subgroup of $S L_{2}(Z)$. There is a congruence subgroup $\Gamma_{1}$ of $\Gamma_{0}$ which leaves each point of $L^{*} / L$ fixed. Let $e_{0}$ be the index of $\Gamma_{1}$ in $\Gamma$. We have,

$$
e_{0} \Theta(z, \varphi)=\sum_{x^{*} \in L^{*} / L} \nu\left(x^{*}\right) \Theta\left(z, f, \Phi, x^{*}\right)
$$

(cf. (1.22)), where we put

$$
v^{(2 k+1) / 4} \Theta\left(z, f, \Phi, x^{*}\right)=\int_{G / \Gamma_{1}} \sum_{x \in L} f_{z}\left(\rho\left(g^{-1}\right)\left(x+x^{*}\right)\right) \Phi(g) d g \quad\left(f_{z}=r\left(\sigma_{z}, Q\right) f\right) .
$$

Take a $\gamma=\left(\begin{array}{ll}a & b \\ c & d\end{array}\right) \in \Gamma(8 D)$. It is easy to see that $\gamma$ satisfies the condition (1.21), $(c / 2) L^{*} \subset L$ and $d x^{*} \equiv x^{*}$ modulo $L$. Hence, by Proposition 1.6,

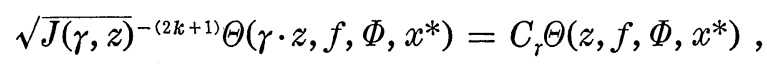

where

$$
C_{\gamma}= \begin{cases}e\left[\frac{a b}{2}\left(x^{*}, x^{*}\right)\right] \quad(c=0), \\ (i)^{-\operatorname{sgn} c} e\left[\frac{a b}{2}\left(x^{*}, x^{*}\right)\right] \varepsilon_{d}^{-3}(i \operatorname{sgn} c)^{3}\left(\frac{2 c}{d}\right)^{3}\left(\frac{D}{-d}\right) & (c \neq 0, d<0), \\ e\left[\frac{a b}{2}\left(x^{*}, x^{*}\right)\right] \varepsilon_{d}^{3}\left(\frac{-2 c}{d}\right)\left(\frac{D}{d}\right) & (c \neq 0, d>0) .\end{cases}
$$

Now it is easy to check that $C_{r}=(c / d) \varepsilon_{d}^{-1}$ for $\gamma \in \Gamma(8 D)$. To prove that $\Theta(z, \varphi)$ vanishes at each cusp of $\Gamma(8 D)$, it is sufficient to show that $J(\gamma, z)^{-(2 k+1)} \Theta(\gamma \cdot z, \varphi)$ vanishes at $+i \infty$ for every $\gamma \in S L_{2}(Z)$. This follows easily from Proposition 1.7 and Proposition 2.1.

5. Let $N$ be a positive integer and $\chi$ be a character modulo $N$. For $\gamma=\left(\begin{array}{ll}a & b \\ c & d\end{array}\right) \in \Gamma_{0}(N)$, set $\chi(\gamma)=\chi(d)$. Then $\chi$ is a character of $\Gamma_{0}(N)$. Set 
$S_{2 k}(N, \chi)=S_{2 k}\left(\Gamma_{0}(N), \chi\right)$. (The definition of $S_{2 k}\left(\Gamma_{0}(N), \chi\right)$ is given in 2). For a rational prime $p$, the Hecke operator $T_{2 k}^{N}(p, \chi)$ is a linear transformation on $S_{2 k}(N, \chi)$ given by

$$
\begin{array}{r}
\left\{T_{2 k}^{N}(p, \chi) \varphi\right\}(z) \\
=p^{2 k-1} \chi(p) \varphi(p z)+p^{-1} \sum_{k=0}^{p-1} \varphi\left(\frac{z+k}{p}\right) \\
(\text { see }(3.5 .7) \text { of [11]). }
\end{array}
$$

For an $N$ divisible by 4 , we denote by $\widetilde{\varsigma}_{2 k+1}(N, \chi)$ the space of holomorphic cusp forms $\psi$ of weight $k+\frac{1}{2}$ and level $N$ which satisfy

$$
\psi(\gamma \cdot z)=\chi(\gamma) j(\gamma, z)^{2 k+1} \psi(z) \quad\left({ }^{\prime} \gamma \in \Gamma_{0}(N)\right) .
$$

For a rational prime $p$, the Hecke operator $T_{2 k+1, \mathrm{x}}^{N}\left(p^{2}\right)$ is a linear transformation on $\mathfrak{S}_{2 k+1}(N, \chi)$ given as follows: If

$$
\begin{gathered}
\psi(z)=\sum_{n=1}^{\infty} a_{n} e[n z] \in \mathbb{S}_{2 k+1}(N, \chi), \\
\left\{T_{2 k+1, \chi}^{N}\left(p^{2}\right) \psi\right\}(z)=\sum_{n=1}^{\infty} b_{n} e[n z],
\end{gathered}
$$

where $b(n)=a\left(p^{2} n\right)+\chi(p)\left(\frac{-1}{p}\right)^{k}\left(\frac{n}{p}\right) p^{k-1} a(n)+\chi\left(p^{2}\right) p^{2 k-1} a\left(n / p^{2}\right), \quad$ (see Theorem 1.7 of [10]).

Let $\mathscr{L}$ be the lattice of integral binary quadratic forms and $\mathscr{L}^{\prime}$ be the sublattice of $\mathscr{L}$ consisting of all forms with even second coefficients. For a positive integer $N$, we set

$$
(x, y)_{N}= \begin{cases}(2 / N)\left(x_{2} y_{2}-2 x_{1} y_{3}-2 x_{3} y_{1}\right) & \text { for } N \text { odd } \\ \left(\frac{1}{2 N}\right)\left(x_{2} y_{2}-2 x_{1} y_{3}-2 x_{3} y_{1}\right) & \text { for } N \text { even }\end{cases}
$$

Put

$$
L_{N}= \begin{cases}N \mathscr{L} & \text { for } N \text { odd }, \\ N \mathscr{L}^{\prime} & \text { for } N \text { even } .\end{cases}
$$

Then $L_{N}^{*}$, the lattice dual to $L_{N}$ with respect to the bilinear form $(,)_{N}$ is given by

$$
L_{N}^{*}= \begin{cases}\frac{1}{4} \mathscr{L}^{\prime} & (N \text { odd }), \\ \mathscr{L} & (N \text { even }) .\end{cases}
$$

Let $\chi$ be a character modulo $N$. Denote by $\nu_{N}^{x}$ the function on $\mathscr{L}$ given 
as follows:

For $N$ odd,

$$
\nu_{N}^{x}(x)= \begin{cases}\chi\left(x_{1}\right) & \text { if } N \mid x_{2} \text { and } N \mid x_{3}, \\ 0 & \text { otherwise }\end{cases}
$$

For $N$ even,

$$
\nu_{N}^{\chi}(x)= \begin{cases}\chi\left(x_{1}\right) & \text { if } 2 N \mid x_{2} \text { and } N \mid x_{3}, \\ 0 & \text { otherwise } .\end{cases}
$$

For $N$ odd, we extend $\nu_{N}^{x}$ to a function on $L_{N}^{*}$ by setting it to be zero outside $\mathscr{L}$. It is easy to see that $\nu_{N}^{x}$ is a function on $L_{N}^{*} / L_{N}$ which satisfies

$$
\nu_{N}^{\chi}(\rho(\gamma) \cdot x)=\chi^{-2}(\gamma) \nu_{N}^{\chi}(x) \quad \text { for any } \gamma \in \Gamma_{0}(N) .
$$

For a $\varphi \in S_{2 k}\left(N, \chi^{2}\right)$, set

$$
\begin{aligned}
\Theta_{\chi}(z, \varphi) & =\Theta\left(z, \varphi, L_{N}, \nu_{N}\right) \\
& =\sum \nu_{N}^{\chi}(x) e\left[\frac{z}{2}(x, x)_{N}\right] \int_{C\left(x, \Gamma_{0}(N)\right)} \varphi(w) x(1,-w)^{k-1} d w,
\end{aligned}
$$

where the summation is taken over all $\Gamma_{0}(N)$-equivalence classes of integral binary quadratic forms with positive discriminant (the definition of $C\left(x, \Gamma_{0}(N)\right)$ is given at the beginning of paragraph 3 of this section). Denote by $\chi^{\prime}$ a character modulo $4 N$ given by

$$
\chi^{\prime}(m)=\chi(m)\left(\frac{N}{m}\right)\left(\frac{-1}{m}\right)^{k} .
$$

Now we state the main theorem in this paper.

THEOREM 2. If

$$
\varphi \in S_{2 k}\left(N, \chi^{2}\right), \text { then } \Theta_{\chi}(z, \varphi) \in \mathbb{S}_{2 k+1}\left(4 N, \chi^{\prime}\right) .
$$

Moreover, if $p$ is an odd prime, then

$$
\Theta_{\chi}\left(z, T_{2 k}^{N}\left(p, \chi^{2}\right) \varphi\right)=T_{2 k+1, \chi^{\prime}}^{4 N}\left(\Theta_{\chi}(z, \varphi)\right)
$$

(this equality holds even for $p=2$, if $N$ is even).

The first part of the theorem is an easy consequence of Theorem 1 Proposition 1.6 and Proposition 1.7. We will devote the remaining part 
of this section to the proof of the second part of the theorem.

6. To prove the second half of Theorem 2, we prepare a few lemmata on lattices in quadratic fields. Let $K=Q(\sqrt{d})$ be the real quadratic field with discriminant $d$. Put $\omega=(d+\sqrt{d}) / 2$. Then 1 and $\omega$ form a $Z$-base of the maximal order $\mathfrak{D}$ of $K$. A rank $2 Z$-module in $K$ is called a lattice if it contains a $Q$-base of $K$. For a lattice $\mathfrak{A}$ in $k$, denote by $\mathfrak{O}(\mathfrak{U})$ the order of $\mathfrak{U}: \mathfrak{N}(\mathfrak{U})=\{k \in K ; k \mathfrak{X} \subset \mathfrak{U}\}$. There exists a unique positive integer $c$ such that $\mathfrak{S}(\mathfrak{U})=\mathfrak{D}_{c}=Z+c \omega Z$. We call $c$ the conductor of $\mathfrak{A}$. For each finite rational prime $p$, put

$$
K_{p}=K \otimes_{Q} Q_{p}, \quad \mathfrak{U}_{p}=\mathfrak{A} \otimes_{Z} Z_{p} .
$$

Then $\mathfrak{U}_{p}$ is a $Z_{p}$-lattice in $K_{p}$ and $\mathfrak{U}_{p}=\mathfrak{S}_{p}$ for almost all $p$. Conversely, if a $Z_{p}$-lattice $\mathfrak{U}_{p}$ in $K_{p}$ is given for each $p$ and if $\mathfrak{N}_{p}=\mathfrak{D}_{p}$ for almost all $p$, there exists a unique lattice $\mathfrak{U}$ in $K$ such that $(\mathfrak{U})_{p}=\mathfrak{U}_{p}$ for all $p$. Set $K_{A}^{\times}=\left\{k \in \prod_{p} K_{p}^{\times} ; k_{p} \in \mathfrak{D}_{p}^{\times}\right.$for almost all $\left.p\right\}$.

For a lattice $\mathfrak{A}$ in $K$ and a $k \in K_{A}^{\times}$, denote by $k \mathfrak{X}$ the lattice given by the equalities $(k \mathfrak{U})_{p}=k_{p} \mathfrak{P}_{p}\left({ }^{\forall} p\right)$.

Thus, $K_{A}^{\times}$operates on the set of lattices in $K$. For a positive integer $c$, denote by $\mathscr{L}(c)$ the set of lattices in $K$ with conductor $c$. It is known (see Proposition 5.4.2. of [11]) that

$$
\mathscr{L}(c)=K_{A}^{\times} \cdot \mathfrak{D}_{c}=\left\{k \cdot \mathfrak{D}_{c} ; k \in K_{A}^{\times}\right\} .
$$

We call two lattices in $K$ equivalent if one is transformed into the other by a multiplication of a suitable element in $K$ with positive norm. Let $\mathfrak{U}$ be a lattice in $K$. Take a $Z$-base $\left\{\omega_{1}, \omega_{2}\right\}$ of $\mathfrak{A}$ which satisfies $\omega_{1} \omega_{2}^{\prime}-$ $\omega_{2} \omega_{1}^{\prime}>0$ (' means the conjugation with respect to $Q$ ). Denote by $\alpha$ the positive generator of the $Z$-ideal $\left(\omega_{1} \omega_{1}^{\prime}, \omega_{1} \omega_{2}^{\prime}+\omega_{1}^{\prime} \omega_{2}, \omega_{2} \omega_{2}^{\prime}\right)$ in $Q$ and put

$$
\mathfrak{U}(X, Y)=\alpha^{-1}\left(\omega_{1} X+\omega_{2} Y\right)\left(\omega_{1}^{\prime} X+\omega_{2}^{\prime} Y\right) .
$$

Then $\mathfrak{A}(X, Y)$ is a primitive integral binary quadratic form (an integral binary quadratic form is said to be primitive if its coefficients have no non-trivial common divisor).

The $S L(2, Z)$-equivalence class of $\mathfrak{Y}(X, Y)$ is uniquely determined by the equivalence class of $\mathfrak{A}$. Now the following lemma is known (see Satz 154 of [4]).

LEMma 2.2. The mapping $\mathfrak{A} \mapsto \mathfrak{A}(X, Y)$ establishes a one to one 
correspondence between the set of equivalence classes of lattices with conductor $c$ in $K$ and the set of $S L(2, Z)$-equivalence classes of primitive integral binary quadratic forms with discriminant $d c^{2}$.

Lemma 2.3. Let $\mathfrak{U}$ be a lattice in $K$ with conductor $c$ and let $p$ be a rational prime. Among $p+1$ sublattices of $\mathfrak{A}$ with index $p$, there are $p-\left(\frac{d}{p}\right)$ (resp. p) with conductor cp and $1+\left(\frac{d}{p}\right)$ (resp. 1) with conductor $c$ (resp. $c / p)$ if $c$ is prime to (resp. divisible by) $p$.

Proof. By (2.20), it is sufficient to prove the lemma under the assumption that $\mathfrak{U}=\mathfrak{D}_{c}$. In this case, the sublattices $\mathfrak{Y}^{(i)}(0 \leq i \leq p)$ of $\mathfrak{U}$ with index $p$ are given by

$$
\mathfrak{U}^{(0)}=Z p+Z c \omega, \quad \mathfrak{X}^{(i)}=(1+c i \omega) Z+p c \omega Z \quad(1 \leqslant i \leqslant p) .
$$

We have

$$
\mathfrak{A}^{(i)}(X, Y)=\left(\alpha_{i} X^{2}+\beta_{i} X Y+\gamma_{i} Y^{2}\right) /\left(\alpha_{i}, \beta_{i}, \gamma_{i}\right),
$$

where

$$
\begin{gathered}
\alpha_{0}=p^{2}, \quad \beta_{0}=p c d, \quad \gamma_{0}=c^{2} d(d-1) / 4 ; \alpha_{i}=1+i c d+i^{2} c^{2} d(d-1) / 4, \\
\beta_{i}=p c d+p i c^{2} d(d-1) / 2 \quad \text { and } \quad \gamma_{i}=p^{2} c^{2} d(d-1) / 4 \quad(1 \leqslant i \leqslant p) .
\end{gathered}
$$

If $c$ is divisible by $p$, it is easy to see that

$$
\left(\alpha_{0}, \beta_{0}, \gamma_{0}\right)=p^{2} \text { and }\left(\alpha_{i}, \beta_{i}, \gamma_{i}\right)=1 \quad \text { for } 1 \leqslant i \leqslant p .
$$

If $c$ is not divisible by $p$, we see, by a straightforward computation, that among $p+1$ integers $\left(\alpha_{i}, \beta_{i}, \gamma_{i}\right) \quad(0 \leqslant i \leqslant p)$, there are exactly $p-\left(\frac{d}{p}\right)$ which are equal to one and $1+\left(\frac{d}{p}\right)$ which are equal to $p$. The lemma now follows from the previous lemma.

Put $\mathfrak{D}_{c}^{\times}=\left\{x \in K ; x \mathfrak{D}_{c}=\mathfrak{O}_{c}, x x^{\prime}>0\right\}$. It is known that $\mathfrak{D}_{c}^{\times} / \pm 1$ is an infinite cyclic group. The next lemma is well-known in the classical theory of binary quadratic forms (see e.g. [1]).

LEMMA 2.4. Let $\varepsilon_{d c^{2}}=t+u c \sqrt{\bar{d}}>1$ be a generator of $\mathfrak{D}_{c}^{\times} / \pm 1$. Let $x(X, Y)=x_{1} X^{2}+x_{2} X Y+x_{3} Y^{2}$ be a primitive integral binary quadratic form with discriminant $d c^{2}$ and set

$$
\gamma_{x}=\left(\begin{array}{cc}
t-x_{2} u & 2 x_{1} u \\
-2 x_{3} u & t+x_{2} u
\end{array}\right)
$$

Then the mapping: $\pm \varepsilon_{d c^{2}}^{m} \rightarrow \pm \gamma_{x}^{m}$, establishes an isomorphism between $\mathfrak{O}_{c}^{\times}$ and the isotropy subgroup of $x$ in $S L_{2}(Z)$. 
It is known that the number of equivalence classes of lattices with conductor $c$ in $K$ is finite. Let $\mathfrak{U}^{1}, \ldots, \mathfrak{A}^{h}$ be a complete set of representatives of these classes. For a rational prime $p$, let $\mathfrak{N}_{0}^{i}, \mathfrak{A}_{1}^{i}, \ldots, \mathfrak{A}_{p}^{i}$ be the $p+1$ sublattices of $\mathfrak{A}^{i}$ with index $p$. By Lemma 2.3, the conductors of $(p+1) h$ lattices $\left\{\mathfrak{U}_{j}^{i} ; 1 \leqslant i \leqslant h, 0 \leqslant j \leqslant p\right\}$ are either $c p$ or $c /(c, p)$.

LEMMA 2.5. (i) For an arbitrarily given lattice with conductor $c p$, there are precisely, $\left[\mathfrak{O}_{c}^{\times}, \mathfrak{O}_{c p}^{\times}\right]$number of lattices among $h(p+1)$ lattices $\left\{\mathfrak{H}_{j}^{i} ; 1 \leqslant i \leqslant h, 0 \leqslant j \leqslant p\right\}$ which are equivalent to the given one.

(ii) Assume $c$ is prime to $p$. For an arbitrarily given lattice with conductor $c$ there are exactly $1+\left(\frac{d}{p}\right)$ number of lattices among $\left\{\mathfrak{U}_{j}^{i}\right\}$ which are equivalent to the given one.

(iii) Assume $c$ is divisible by $p$. For an arbitrarily given lattice with conductor $c / p$, there are exactly $p /\left[\mathfrak{O}_{c / p}^{\times}, \mathfrak{N}_{c}^{\times}\right]\left(\operatorname{resp} .\left\{p-\left(\frac{d}{p}\right)\right\} /\left[\mathfrak{O}_{c / p}^{\times}, \mathfrak{N}_{c}^{\times}\right]\right)$ number of lattices among $\left\{\mathfrak{Q}_{j}^{i}\right\}$ which are equivalent to the given one if $c$ is divisible (resp. not divisible) by $p^{2}$.

Proof. Set $K^{+}=\left\{k \in K ; k k^{\prime}>0\right\}$. We have

$$
\mathscr{L}(c)=\bigcup_{k \in K+10_{c}^{\times}} \bigcup_{i=1}^{h} k \cdot \mathfrak{A}^{i} \quad \text { (disjoint union), }
$$

where $\mathscr{L}(c)$ is the set of lattices with conductor $c$. Hence, if $c$ is prime to $p$, it follows from Lemma 2.3 that

$$
\bigcup_{k \in K^{+} / 0_{c}^{\times}} \bigcup_{i=1}^{h} \bigcup_{j=0}^{p} k \cdot \mathfrak{Y}_{j}^{i}=\mathscr{L}(c p)+\left\{1+\left(\frac{d}{p}\right)\right\} \mathscr{L}(c) .
$$

Namely, each lattice with conductor $c p$ (resp. $c$ ) appears exactly once (resp. $1+\left(\frac{d}{p}\right)$ times) among $\left\{k \cdot \mathscr{O}_{j}^{i} ; k \in K^{+} / \mathfrak{D}_{c}^{\times}, 1 \leq i \leq h, 0 \leqslant j \leqslant p\right\}$.

Let $\mathscr{L}^{1}, \ldots, \mathscr{L}^{h(c p)}$ be a complete set of representatices of lattices with conductor $c p$. Then

$$
\mathscr{L}(c p)=\bigcup_{k \in K+10_{c p}^{\times}} \bigcup_{i=0}^{h(c p)} k \cdot \mathscr{L}^{(i)} \quad \text { (disjoint union). }
$$

Thus, lemma is now obvious for $c$ prime to $p$. If $c$ is divisible by $p$, it follows from Lemma 2.3 that the left hand side of (2.22) is equal to 
$\mathscr{L}(c p)+p \mathscr{L}(c / p)$ or $\mathscr{L}(c p)+\left\{p-\left(\frac{d}{p}\right)\right\} \mathscr{L}(c / p)$ according as $c$ is divisible by $p^{2}$ or not. Hence the lemma is checked in a similar manner.

For a positive integer $N$, we denote by $L(N)$ the subset of primitive integral binary quadratic forms given as follows:

$$
L(N)= \begin{cases}\left\{x ; N\left|x_{2}, N\right| x_{3} ;\left(x_{1}, x_{2}, x_{3}\right)=1\right\} & (N \text { odd }), \\ \left\{x ; 2 N\left|x_{2}, N\right| x_{3} ;\left(x_{1}, x_{2}, x_{3}\right)=1\right\} & (N \text { even }) .\end{cases}
$$

It is obvious that $L(N)$ is invariant under the action of $\Gamma_{0}(N)$.

LEMMA 2.6. Two $S L(2, Z)$-equivalent points in $L(N)$ are $\Gamma_{0}(N)$-equivalent. For $N$ odd (resp. even), each primitive integral binary quadratic form with discriminant divisible by $N$ (resp. $4 N$ ) is $S L(2, Z$ )-equivalent to a form in $L(N)$.

Proof. The first half of the lemma follows easily from (2.2). To prove the second half, we may assume that $N=p^{m}(m \geqslant 1)$ is a power of a prime number $p$. We will prove the lemma for $p=2$. For odd $p$, the proof is similar but simpler. Let $x=x_{1} X^{2}+x_{2} X Y+x_{3} Y^{2}$ be a primitive integral quadratic form with discriminant divisible by $2^{m+2}$. Transforming $x$ by a suitable element of $S L(2, Z)$ if necessary, we may assume that $x_{1}$ is odd and $x_{2}$ is a multiple of 4 . Then $x_{3}$ is even. Thus, for $m=1$, the lemma is valid. Next we assume (making use of the induction with respect to $m$ ) that $m \geqslant 2$ and there exists a form $x^{\prime}(X, Y)=x_{1}^{\prime} X^{2}+x_{2}^{\prime} X Y+x_{3}^{\prime} Y^{2}$ which is $S L(2, Z)$-equivalent to $x$ and has a second coefficient $x_{2}^{\prime}$ divisible by $2^{m}$ and has a third coefficient $x_{3}^{\prime}$ divisible by $2^{m-1}$.

For a suitable integer $c, x_{2}^{\prime}+2^{m} \cdot c x_{1}^{\prime}$ is divisible by $2^{m+1}$. Then

$$
\rho\left(\left[\begin{array}{ll}
1 & \\
2^{m-1} c & 1
\end{array}\right]\right) \cdot x^{\prime} \text { is in } L\left(2^{m}\right) \text {. }
$$

The next lemma is an easy consequence of Lemma 2.4 and the definition of $C\left(x, \Gamma_{0}(N)\right)$ given at the beginning of paragraph 3 of this section.

LEMMA 2.7. Take an integral binary quadratic form $x \in L(N)$.

(i) If the discriminant of $x$ is not a square, $C\left(x, \Gamma_{0}(N)\right)$ is a rectifiable curve in $\mathfrak{K}$ from $w$ to $\gamma_{x} \cdot w$, where $w$ is any point of $\mathfrak{F}_{\mathrm{C}}$, and $\gamma_{x}$ is given by (2.21).

(ii) If the discriminant of $x$ is the square of a positive number 
$m, C\left(x, \Gamma_{0}(N)\right)$ is the geodesic line from $\left(x_{2}+m\right) / 2 x_{3}$ to $\left(x_{2}-m\right) / 2 x_{3}$ (if $x_{3}=0$, we understand that $C\left(x, \Gamma_{0}(N)\right)$ is the geodesic line from $+i \infty$ (resp. $\left.x_{1} / x_{2}\right)$ to $x_{1} / x_{2}($ resp. $+i \infty)$ for $x_{2}>0\left(\right.$ resp. $\left.x_{2}<0\right)$ ).

(iii) If $l$ is a positive integer prime to $N, C\left(l x, \Gamma_{0}(N)\right)=C\left(x, \Gamma_{0}(N)\right)$ for any $x \in L(N)$.

For a $\varphi \in S_{2 k}\left(N, \chi^{2}\right)$ and a positive integer $n$, set

$$
\alpha_{N}(n, \varphi)=\sum \nu_{N}^{x}(x) \int_{C(x, N)} \varphi(z) x(1,-z)^{k-1} d z,
$$

where $\nu_{N}^{x}(x)=\chi\left(x_{1}\right), C(x, N)=C\left(x, \Gamma_{0}(N)\right)$ and the summation is taken over all $\Gamma_{0}(N)$-equivalence classes of forms in $L(N)$ with discriminant $N n$ (resp. $4 N n$ ) for $N$ odd (resp. even). For $N$ odd (resp. even) denote by $d_{n}$ the discriminant of the number field $Q(\sqrt{n N})$ (resp. $Q(\sqrt{4 n N})$ ) and put $n N=d_{n} c_{n}^{2}$ (resp. $4 n N=d_{n} c_{n}^{2}$ ). Then $c_{n}$ is a positive integer or a positive half-integer. In the latter case (which can occur only for $N$ odd), $\alpha_{N}(n, \varphi)=0$ for every $\varphi \in S_{2 k}\left(N, \chi^{2}\right)$. Now we assume that $c_{n}$ is an integer.

LEMMA 2.8. Notations being as above, one has

$$
\begin{aligned}
\alpha_{N}\left(n, T_{2 k}^{N}\left(p, \chi^{2}\right) \varphi\right) & \begin{cases}\alpha_{N}\left(n p^{2}, \varphi\right)+p^{k-1}\left(1+\left(\frac{d_{n}}{p}\right)\right) \chi(p) \alpha_{N}(n, \varphi) & \text { if } \quad\left(p, c_{n}\right)=1, \\
\alpha_{N}\left(n p^{2}, \varphi\right)+p^{2 k-2}\left(p-\left(\frac{d_{n}}{p}\right)\right) \chi\left(p^{2}\right) \alpha_{N}\left(n / p^{2}, \varphi\right) & \text { if }\left(p^{2}, c_{n}\right)=p, \\
\alpha_{N}\left(n p^{2}, \varphi\right)+p^{2 k-1} \chi\left(p^{2}\right) \alpha_{N}\left(n / p^{2}, \varphi\right) & \text { if }\left(p^{2}, c_{n}\right)=p^{2},\end{cases}
\end{aligned}
$$

where we put $\alpha_{N}\left(n / p^{2}, \varphi\right)=0$ for $n$ not divisible by $p^{2}$.

Proof. Assume $d_{n}>1$ and put $K=Q\left(\sqrt{d_{n}}\right)$. Let $x^{1}, x^{2}, \cdots, x^{h}$ be a complete set of representatives of $\Gamma_{0}(N)$-equivalence classes of forms in $L(N)$ with discriminant $d_{n} c_{n}^{2}$. Set

$$
x^{i}(X, Y)=x_{1}^{i} X^{2}+x_{2}^{i} X Y+x_{3}^{i} Y^{2} \quad(1 \leqslant i \leqslant h) .
$$

Set $\gamma_{i}=\gamma_{x^{i}}(1 \leqslant i \leqslant h)$ (see (2.21)). Then we have, by Lemma 2.7 and (2.24),

$$
\begin{aligned}
& \alpha_{N}\left(n, T_{2 k}^{N}\left(p, \chi^{2}\right) \varphi\right) \\
& \quad=\sum_{i=1}^{h} \nu_{N}^{\chi}\left(x^{i}\right) \int_{w}^{r_{i} \cdot w} T_{2 k}^{N}\left(p, \chi^{2}\right) \varphi(z) x^{i}(1,-z)^{k-1} d z .
\end{aligned}
$$


Put $\omega_{1}^{i}=\left(x_{2}^{i}+c_{n} \sqrt{d_{n}}\right) / 2$ and $\omega_{2}^{i}=x_{3}^{i}$ and denote by $\mathfrak{Y}^{i}$ the lattice in $K$ generated by $\omega_{1}^{i}$ and $\omega_{2}^{i}$. It is easy to see that $\mathfrak{A}^{i}(X, Y)=x^{i}(X, Y)$ $(1 \leqslant i \leqslant h)$. It follows from Lemma 2.2 and Lemma 2.6 that $\left\{\mathfrak{U}^{1}, \mathfrak{U}^{2}, \ldots \mathfrak{A}^{h}\right\}$ forms a complete set of representatives of equivalence classes of lattices with conductor $c_{n}$ in $K$.

Put

$$
\begin{gathered}
x^{i, 0}=\rho\left(\left[\begin{array}{ll}
p & \\
& 1
\end{array}\right]\right) \cdot x^{i}, x^{i, j}=\rho\left(\left[\begin{array}{ll}
1 & j \\
& p
\end{array}\right]\right) \cdot x^{i} \quad(1 \leqslant j \leqslant p), \\
\gamma_{i 0}=\left(\begin{array}{ll}
p & \\
& 1
\end{array}\right) \gamma_{i}\left(\begin{array}{ll}
p & \\
& 1
\end{array}\right)^{-1} \text { and } \quad \gamma_{i, j}=\left(\begin{array}{ll}
1 & j \\
& p
\end{array}\right) \gamma_{i}\left(\begin{array}{ll}
1 & j \\
& p
\end{array}\right)^{-1} \quad(1 \leqslant j \leqslant p) .
\end{gathered}
$$

Further, set $e_{1}=\left[\mathfrak{D}_{c}^{\times}, \mathfrak{D}_{c p}^{\times}\right]$and $e_{2}=\left[\mathfrak{D}_{c /(c, p)}^{\times}, \mathfrak{D}_{c}^{\times}\right]$. We note that $\nu_{N}^{\times}\left(x^{i, 0}\right)=$ $\chi\left(p^{2}\right) \nu_{N}^{x}\left(x^{i}\right)$ and $\nu_{N}^{x}\left(x^{i, j}\right)=\nu_{N}^{x}\left(x^{i}\right)(1 \leqslant j \leqslant p)$. It follows from (2.18) that

$$
\begin{aligned}
\nu_{N}^{\chi}\left(x^{i}\right) \int_{w}^{\gamma_{i} \cdot w} & T_{2 k}^{N}\left(p, \chi^{2}\right) \varphi(z) x^{i}(1,-z)^{k-1} d z \\
& =e_{1}^{-1} \sum_{j=0}^{p} \nu_{N}^{\chi}\left(x^{i, j}\right) \int_{w}^{\gamma_{i j}^{e 1} \cdot w} \varphi(z) x^{i, j}(1,-z)^{k-1} d z .
\end{aligned}
$$

Set

$$
\mathfrak{A}^{i, 0}=p \omega_{1}^{i} Z+\omega_{2}^{i} Z \text { and } \mathfrak{U}^{i, j}=\left(\omega_{1}^{i}+j \omega_{2}^{i}\right) Z+p \omega_{2}^{i} Z \quad(1 \leqslant j \leqslant p) .
$$

Then $\mathfrak{U}^{i, 0}, \mathfrak{U}^{i, 1}, \ldots, \mathfrak{U}^{i, p}$ are mutually distinct sublattices of $\mathfrak{Y}^{i}$ with index p. Put $y^{i, j}(X, Y)=\mathfrak{Q}^{i, j}(X, Y)$. Then $x^{i, j}=y^{i, j}$ or $p y^{i, j}$ or $p^{2} y^{i, j}$ according as the conductor of $\mathfrak{Q}^{i, j}$ is $c p$ or $c$ or $c / p$. It is easy to check that $\gamma_{i_{j}}^{e_{1}}$ is $\gamma_{\left(y^{i j}\right)}$ or $\gamma_{\left(y_{i j}\right)}^{e_{1}}$ or $\gamma_{\left(y^{i j}\right)}^{e_{1} e_{2}}$ according as the conductor of $\mathfrak{Q}^{i j}$ is $c p$ or $c$ or $c / p$. Lemma 2.8 is now an easy consequence of Lemma 2.5, Lemma 2.7 and Lemma 2.2. If $d_{n}=1$, the proof is similar and much simpler.

Set $\Theta(z, \varphi)=\sum_{n \geq 1} a(n) e[n z]$ and

$$
\Theta\left(z, T_{2 k}^{N}\left(p, \chi^{2}\right) \varphi\right)=\sum_{n \geq 1} b(n) e[n z]
$$

$(\Theta(z, \varphi)$ is given by $(2.19))$.

LEMMA 2.9. Let $d_{n}$ be the discriminant of $Q(\sqrt{N n})$. Set $N n=d_{n} c_{n}^{2}$ (resp. $4 N n=d_{n} c_{n}^{2}$ ) for $N$ odd (resp. even). If $c_{n}$ is not an integer, $a(n)=b(n)=0$. If $c_{n}$ is an integer,

$$
b(n)=a\left(n p^{2}\right)+p^{k-1} \chi(p)\left(\frac{N n}{p}\right) a(n)+p^{2 k-1} \chi\left(p^{2}\right) a\left(n / p^{2}\right),
$$


where we understand that $a\left(n / p^{2}\right)=0$ if $n / p^{2}$ is not an integer.

Proof. If follows from (2.19) and (2.24) that

$$
a(n)=\sum \chi(m) m^{k-1} \alpha_{N}\left(n / m^{2}, \varphi\right)
$$

and

$$
b(n)=\sum \chi(m) m^{k-1} \alpha_{N}\left(n / m^{2}, T_{2 k}^{N}\left(p, \chi^{2}\right) \varphi\right),
$$

where the summation is taken over all positive divisors of $c_{n}$ which are prime to $N$. If $c_{n}$ is not an integer, it is clear that $a(n)=b(n)=0$. Assume that $c_{n}$ is an integer. If $p$ is a factor of $N$, we have, by Lemma 2.8 ,

$$
b(n)=\sum \chi(m) m^{k-1} \alpha_{N}\left(n p^{2} / m^{2}, \varphi\right)=a\left(n p^{2}\right) .
$$

Next we assume that $p$ is prime to both $N$ and $c_{n}$. Then Lemma 2.6 implies

$$
\begin{aligned}
b(n) & =\sum_{m \mid c_{n}} \chi(m) m^{k-1}\left\{\alpha_{N}\left(n p^{2} / m^{2}, \varphi\right)+p^{k-1}\left(1+\left(\frac{d_{n}}{p}\right)\right) \chi(p) \alpha_{N}\left(n / m^{2}, \varphi\right)\right\} \\
& =a\left(n p^{2}\right)+p^{k-1} \chi(p)\left(\frac{N n}{p}\right) a(n) .
\end{aligned}
$$

Finally assume that $p$ is prime to $N$ but is a factor of $c_{n}$. Set $c_{n}=$ $c_{n}^{\prime} p^{l}$, where $c_{n}^{\prime}$ is prime to $p$. We have

$$
b(n)=\sum \chi\left(m^{\prime} p^{i}\right)\left(p^{i} m^{\prime}\right)^{k-1} \alpha_{N}\left(n / m^{\prime 2} p^{2 i}, T_{2 k}^{N}\left(p, \chi^{2}\right) \varphi\right),
$$

where the summation is taken over all positive divisors $m^{\prime}$ of $c_{n}^{\prime}$ and over all non-negative integers $i$ not exceeding $l$.

Now Lemma 2.8 shows that

$$
\begin{aligned}
& \alpha_{N}\left(n / m^{\prime 2} p^{2 i}, T_{2 k}^{N}\left(p, \chi^{2}\right) \varphi\right) \\
& =\left\{\begin{array}{lr}
\alpha_{N}\left(n p^{2} / m^{\prime 2} p^{2 i}, \varphi\right)+p^{2 k-1} \chi\left(p^{2}\right) \alpha_{N}\left(n / m^{\prime 2} p^{2 i+2}, \varphi\right) & (0 \leq i \leq l-2), \\
\alpha_{N}\left(n p^{2} / m^{\prime 2} p^{2 i}, \varphi\right)+p^{2 k-2} \chi\left(p^{2}\right)\left(p-\left(\frac{d_{n}}{p}\right)\right) \alpha_{N}\left(n / m^{\prime 2} p^{2 i+2}, \varphi\right) & (i=l-1), \\
\alpha_{N}\left(n p^{2} / m^{\prime 2} p^{2 l}, \varphi\right)+p^{k-1}\left(1+\left(\frac{d_{n}}{p}\right)\right) \chi(p) \alpha_{N}\left(n / m^{\prime 2} p^{2 l}, \varphi\right) & (i=l) .
\end{array}\right.
\end{aligned}
$$

Thus, $b(n)=a\left(n p^{2}\right)+p^{2 k-1} \chi\left(p^{2}\right) a\left(n / p^{2}\right)$.

Lemma 2.9 has been proved.

The second half of Theorem 2 now follows immediately from Lemma 2.7 and the definition of $T_{2 k+1, x^{\prime}}^{4 N}\left(p^{2}\right)$. 


\section{§ 3 Numerical examples}

In this section, we present three numerical examples of Theorem 2.

1. Set $k=6, N=1, \chi=1$. In this case, the space $S_{12}\left(1, \chi^{2}\right)$ is one dimensional and spanned by the function

$$
\Delta(z)=e[z] \prod_{n=1}^{\infty}(1-e[n z])^{24}=\sum_{n=1}^{\infty} \tau(n) e[n z] .
$$

Set $\Theta(z, \Delta)=\Theta(z, 1,1, \Delta)=\sum_{n \geq 1} b_{n} e[n z]$. (see (2.19)). Then, we have

$$
b_{n}=\sum_{x} \int_{C\left(x, \Gamma_{0}(1)\right)} \Delta(z)\left(x_{1}-z x_{2}+x_{3} z^{2}\right)^{5} d z,
$$

where the summation is taken over all $S L(2, Z)$-equivalence classes of integral binary quadratic forms with discriminant $n$. We note that any integral binary quadratic form with discriminant one is $S L(2, Z)$-equivalent to the form $X Y$. Hence, it follows from Lemma 2.7 that

$$
b_{1}=-\int_{0}^{\infty} \Delta(i t)(-i t)^{5} d(i t)=-D(6),
$$

where $D(s)=(2 \pi)^{-s} \Gamma(s) \sum_{n=1}^{\infty} \tau(n) n^{-s}$.

Since $D(s)$ does not vanish on the real line, $b_{1} \neq 0$. By Theorem 2 $\Theta(z, \Delta) \in \mathbb{S}_{13}(4,1)$. Since $\theta(z)=\prod_{n=1}^{\infty}\left(1-q^{2 n}\right)\left(1+q^{2 n-1}\right)^{2}(q=e[z]), \theta(z)$ has not a zero on the upper half plane. Hence, $\psi(z)=\Theta(z, \Delta) / \theta(z)$ is a holomorphic function on the upper half plane which satisfies $\psi(\gamma \cdot z)=$ $(c z+d)^{6} \psi(z)$ for every $\gamma \in \Gamma_{0}(4)$. Now, $\Gamma_{0}(4)$ has three inequivalent cusps, $i \infty, 0$ and $\frac{1}{2}$. It follows from the transformation formula for $\theta(z)$ that it is finite and does not vanish at $i \infty$ and 0 , and that it has a zero of order $1 / 4$ at $\frac{1}{2}$ with respect to a uniformizing parameter $t=$ $e[(-z+1) /(-2 z+1)]$. Furthermore, $\Theta(z, \Delta)$ vanishes at all the cusps of $\Gamma_{0}(4)$ and the order of zero at $1 / 2$ with respect to $t$ is not smaller than 1/4. Thus, $\psi(z)$ is a holomorphic integral form of weight 6 with respect to $\Gamma_{0}(4)$ which vanishes at $i \infty$ and at 0 . It is known that the space of such forms is spanned by

$$
\sqrt{\Delta(2 z)}=q \prod_{n=1}^{\infty}\left(1-q^{2 n}\right)^{12}=q-12 q^{3}+\cdots
$$

and by

$$
E(z)=\sum_{m, m_{1}=1}^{\infty} m^{5}(-1)^{m} q^{m\left(2 m_{1}-1\right)}=-q+32 q^{2}+\cdots
$$


Hence $\Theta(z, \Delta)$ is expressed as a linear combination of $\theta(z) \sqrt{\Delta(2 z)}=2+$ $2 q^{2}+\cdots$ and of $\theta\left((z) E(z)=-q+30 q^{2}+\cdots\right.$. Since $b_{1}=-D(6)$ and $b_{2}=0$ (see (3.2)), we have

$$
\Theta(z, \Delta)=\frac{D(6)}{16}(E(z)-15 \sqrt{\Delta(2 z)}) \theta(z) .
$$

Combining (3.2) and (3.3) we obtain series of identities which may be of some interest. For example, let $d$ be a discriminant of a real quadratic field with class number 1 and let $\varepsilon>1$ be the fundamental unit of $Q(\sqrt{d})$. Set $t+u \sqrt{d}=\varepsilon$ or $\varepsilon^{2}$ according as the norm of $\varepsilon$ is 1 or -1 . Assume $d$ is divisible by 4 and set

$$
\{E(z)-15 \sqrt{\Delta(2 z)}\} \theta(z)=\sum_{n=1}^{\infty} \beta_{n} q^{n}
$$

Then

$$
\int_{z=t / 2 u}^{z=(t / 2 u)+i \infty} \Delta(z)\left(\frac{d}{4}-z^{2}\right)^{5} d z=-\frac{\beta_{d}}{16} D(6) \quad \text { or } \quad-\frac{\beta_{d}}{32} D(6)
$$

according as the norm of $\varepsilon$ is -1 or 1 . In fact, under our assumptions, a complete set of representatives of $\Gamma_{0}(1)$-equivalence classes of integral binary quadratic forms with discriminant $d$ is given by $\left\{ \pm\left((d / 4) X^{2}-Y^{2}\right)\right\}$ or $\left\{(d / 4) X^{2}-Y^{2}\right\}$ according as the norm of $\varepsilon$ is 1 or -1. Furthermore, for $x(X, Y)=(d / 4) X^{2}-Y^{2}$, the integral path $C\left(x, \Gamma_{0}(1)\right)$ in (3.2) is any rectifiable curve from $w$ to $(t w+(d / 2) u) /(2 u w+t)$, where $w$ is any point on the upper half plane. Letting $w \rightarrow+i \infty$ in (3.2) and making use of (3.3), we obtain (3.4) (Cf. Manin [14]).

2. In the following two examples, we are interested in the case where $k=1$ and $\chi$ is a quadratic character modulo $N\left(\chi^{2}=1\right)$. Take a $\varphi \in S_{2}\left(\Gamma_{0}(N), \chi^{2}\right)$. For $N$ odd, we have, by (2.19),

$$
\Theta_{\chi}(z, \varphi)=\sum \chi\left(m x_{1}\right) e\left[\frac{m^{2} z}{N}\left(x_{2}^{2}-4 x_{1} x_{3}\right)\right] \int_{C\left(x, \Gamma_{0}(N)\right)} \varphi(z) d z,
$$

where the summation is taken over all positive integers $m$ and over all $\Gamma_{0}(N)$-equivalence classes of primitive integral binary quadratic forms $x(X, Y)=x_{1} X^{2}+x_{2} X Y+x_{3} Y^{2}$ with positive discriminant and with $x_{2}$ and $x_{3}$ divisible by $N$.

For $N$ even, 


$$
\Theta_{\chi}(z, \varphi)=\sum \chi\left(m x_{1}\right) e\left[\frac{m^{2} z}{4 N}\left(x_{2}^{2}-4 x_{1} x_{3}\right)\right] \int_{C\left(x, \Gamma_{0}(N)\right)} \varphi(z) d z
$$

where the summation is taken over all positive integers $m$ and over all $\Gamma_{0}(N)$-equivalence classes of primitive integral binary quadratic forms with positive discriminant and with $x_{2}$ divisible by $2 N$ and with $x_{3}$ divisible by $N$.

In both cases, it follows from Theorem 2 that

$$
\Theta_{\chi}(z, \varphi) \in \widetilde{S}_{3}\left(4 N, \chi^{\prime}\right), \text { where } \quad \chi^{\prime}(d)=\chi(d)\left(\frac{-1}{d}\right)\left(\frac{N}{d}\right)
$$

Assume that the discriminant of $x(x \in L(N))$ is not a square. Then $C\left(x, \Gamma_{0}(N)\right)$ is a rectifiable curve from $z$ to $\gamma_{x} \cdot z(z \in \mathfrak{S})$, where $\gamma_{x}$ is given by (2.21).

Denote by $X(N)$ the Riemann surface $\Gamma_{0}(N) \backslash \mathscr{S} \cup(i \infty) \cup Q$.

The image of $C\left(x, \Gamma_{0}(N)\right)$ by the natural projection of $\mathscr{S}_{\mathcal{S}}$ into $X(N)$ is a cycle on $X(N)$ which we denote by $\tilde{\gamma}_{x}$. The integral $\int_{C\left(x, \Gamma_{0}(N)\right)} \varphi(z) d z$ is the period of the holomorphic differential form $\varphi(z) d z$ on $X(N)$ along the cycle $\tilde{\gamma}_{x}$. Hence, it is expressed by a linear combination of fundamental periods of $\varphi(z) d z$ on $X(N)$ with coefficients in $Z$. A description of $H_{1}(X(N), Z)$ after Manin [6], which we will recall in the next paragraph, enables us to compute these coefficients in finite steps.

3. The set of inequivalent cusps of $\Gamma_{0}(N)$ is, by definition, the set of $\Gamma_{0}(N)$-orbits in $Q \cup i \infty$. Denote by $\Pi(N)$ the set which consists of pairs of the form $\left[\delta ; a \bmod .\left(\delta, N \delta^{-1}\right)\right]$, here $\delta$ runs through all positive divisors of $N$ and the second coordinate of the pair runs through all invertible classes of residues modulo the greatest common divisor of $\delta$ and $N \delta^{-1}$. If $\left(\delta, N \delta^{-1}\right)=1$, we sometimes put simply 1 in place of the second coordinate. For $r=(u / v \delta) \in \boldsymbol{Q}$, where $\delta \mid N, u, v \in Z,(u, v \delta)=$ $\left(v, N \delta^{-1}\right)=1$, set $c(r)=\left[\delta ; u v \bmod \left(\delta, N \delta^{-1}\right)\right] . \quad$ Further set $c(i \infty)=[N ; 1]$. Then the mapping $c$ establishes one to one correspondence between the set of inequivalent cusps of $\Gamma_{0}(N)$ and the set $\Pi(N)$. We identify these two sets. For each pair $\left(r_{1}, r_{2}\right)$ of two elements of $Q \cup i \infty$, we denote by $\left\{r_{1}, r_{2}\right\}_{N}$ the image, by the natural projection from $\mathfrak{F} \cup \boldsymbol{Q} \cup i \infty$ into $X(N)$, of the geodesic from $r_{1}$ to $r_{2}$. Let $\tilde{c}=c \bmod . N, \tilde{d}=d \bmod . N$ be two residue classes mod. $N$ which are represented by relatively prime 
integers $c$ and $d$. We call two such pairs equivalent if one goes to the other by multiplication of an invertible residue class modulo $N$. The set of these classes $\tilde{c}: \tilde{d}$ is $P^{1}(Z /(N))$, the projective line over $Z /(N)$. For $\tilde{c}: \tilde{d} \in P^{1}(Z /(N))$, set

$$
\xi(\tilde{c}: \tilde{d})=\left\{\frac{b}{d}, \frac{a}{c}\right\}_{N},
$$

where $a, b, c, d$ are integers with the conditions $a d-b c=1, \tilde{c}=c \bmod . N$ and $\tilde{d}=d \bmod . N$. Then $\xi$ is a well-defined map from $P^{1}(Z /(N))$ into the set of chains on $X(N)$. Further, set

$$
\begin{aligned}
\partial(\tilde{c}: \tilde{d})= & {\left[\delta_{1} ; \frac{c}{\delta_{1}} d^{-1}, \bmod .\left(\delta_{1}, N \delta_{1}^{-1}\right)\right] } \\
& -\left[\delta_{2} ;-\frac{d}{\delta_{2}} c^{-1}, \bmod .\left(\delta_{2}, N \delta_{2}^{-1}\right)\right],
\end{aligned}
$$

where $\delta_{1}=(c, N), \delta_{2}=(d, N)$ (the right hand side is understood to be an element of free abelian group generated by elements of $\Pi(N)$ ).

Lemma 3.1. (See Theorem 2.7 of Manin [6]). a) Construct the maximal torsion-free abelian group $\tilde{H}(N)$ generated by the symbols $(\tilde{c}: \tilde{d})$, one for each $\tilde{c}: \tilde{d} \in P^{1}(Z /(N))$ with the relations $(\tilde{c}: \tilde{d})+(-\tilde{d}: \tilde{c})=0$, $(\tilde{c}: \tilde{d})+((\tilde{c}-\tilde{d}): \tilde{c})+(-\tilde{d}:(\tilde{c}-\tilde{d}))=0$. Further let $H(N)$ designate the subgroup in it which is the kernel of the boundary homomorphism (3.10). Then the map $\xi$, given by (3.9), induces an isomorphism $\xi: H(N)$ $\stackrel{\sim}{\longrightarrow} H_{1}(X(N), Z)$.

b) For a $g=\left(\begin{array}{ll}a & b \\ c & d\end{array}\right) \in \Gamma_{0}(N)$, let

$$
\frac{b}{d}=p_{0}+\frac{1}{p_{1}}+\frac{1}{p_{2}}+\cdots+\begin{gathered}
1 \\
p_{n}
\end{gathered}
$$

$\left(p_{0} \in Z, p_{1}, \cdots, p_{n}\right.$ positive integers) be the expansion of $b / d$ into finite continued fractions. Set $d_{-1}=0, d_{0}=1$ and $d_{k}=p_{k} d_{k-1}+d_{k-2}(k=1$, $\cdots, n)$. Then $\sum_{k=1}^{n}\left((-1)^{k-1} \tilde{d}_{k}: \tilde{d}_{k-1}\right) \in H(N)$ is mapped, by $\xi$, into the integral 1-homology of $H_{1}(X(N), Z)$ represented by the cycle $\{0, g \cdot 0\}_{N}$.

EXAMPLES OF LEMMA 3. (i) For $N=11$, the following relations betweeen elements of $\tilde{H}(11)$ hold. 
$\tilde{10}: \tilde{1}=\tilde{1}: \tilde{1}=0, \quad \tilde{1}: \tilde{0}=-(\tilde{0}: \tilde{1}), \quad \tilde{2}: \tilde{1}=-(\tilde{6}: \tilde{1})=-(\tilde{5}: \tilde{1})=\tilde{9}: \tilde{1}$,

$\tilde{3}: \tilde{1}=-(\tilde{7}: \tilde{1}), \quad \tilde{8}: \tilde{1}=-(\tilde{4}: \tilde{1})=(\tilde{2}: \tilde{1})-(\tilde{3}: \tilde{1})$.

Thus, $\tilde{H}(11)$ is a free abelian group generated by $(\tilde{1}: \tilde{0}),(\tilde{2}: \tilde{1})$ and $(\tilde{3}: \tilde{1})$. By $(3.10), \partial(\tilde{1}: \tilde{0})=[1 ; 1]-[11 ; 1], \partial(\tilde{2}: \tilde{1})=\partial(\tilde{3}: \tilde{1})=0$. Hence, $H(11)$ is a free abelian group generated by $\tilde{2}: \tilde{1}$ and by $\tilde{3}: \tilde{1}$. It follows that $\xi(\widetilde{2}: \tilde{1})=\left\{0, \frac{1}{2}\right\}_{11}$ and $\xi(\tilde{3}: \tilde{1})=\left\{0, \frac{1}{3}\right\}_{11}$ form a base of $H_{1}(X(11), Z)$.

(ii) For $N=14$, relations between elements of $\tilde{H}(14)$ are as follows :

$$
\begin{aligned}
& \tilde{1}: \tilde{1}=\widetilde{13}: \tilde{1}=0, \quad \tilde{0}: \tilde{1}=-(\tilde{1}: \tilde{0}) \text {, } \\
& \tilde{2}: \tilde{1}=-(\widetilde{1}: \widetilde{2})=-(\widetilde{13}: \widetilde{2})=\widetilde{12}: \tilde{1}, \quad \tilde{3}: \tilde{1}=-(\widetilde{9}: \tilde{1}) \text {, } \\
& \tilde{4}: \tilde{1}=-(\tilde{3}: \tilde{2}), \quad \tilde{5}: \tilde{1}=-(\widetilde{1}: \tilde{1})=(\tilde{4}: \tilde{1})-(\tilde{2}: \tilde{1}) \text {, } \\
& \tilde{6}: \tilde{1}=-(\tilde{9}: \tilde{2})=(\tilde{4}: \tilde{1})-(\tilde{3}: \tilde{1}), \quad(\tilde{7}: \tilde{1})=-(\tilde{1}: \tilde{7}) \text {, } \\
& \tilde{8}: \tilde{1}=-(\tilde{5}: \tilde{2})=(\tilde{4}: \tilde{1})-(\tilde{3}: \tilde{1}) \text {, } \\
& \widetilde{10}: \tilde{1}=-(\widetilde{1}: \widetilde{2})=(\tilde{2}: \tilde{1})-(\tilde{3}: \tilde{1}) \text {, } \\
& \tilde{7}: \tilde{2}=-(\tilde{2}: \tilde{7})=(\tilde{7}: \tilde{1})-(\tilde{4}: \tilde{1})+(\tilde{3}: \tilde{1}) .
\end{aligned}
$$

Hence, $\tilde{H}(14)$ is generated by $\tilde{0}: \tilde{1}, \tilde{2}: \tilde{1}, \tilde{3}: \tilde{1}, \tilde{4}: \tilde{1}$ and by $\tilde{7}: \tilde{1}$. Furthermore, $\partial(\widetilde{0}: \tilde{1})=[14 ; 1]-[1,1], \partial(\tilde{2}: \tilde{1})=[2 ; 1]-[1 ; 1], \partial(\tilde{3}: \tilde{1})=0, \partial(\tilde{4}: \tilde{1})$ $=[2 ; 1]-[1 ; 1], \partial(\tilde{7}: \tilde{1})=[7 ; 1]-[1 ; 1]$. Thus, $H(14)$ is a free abelian group generated by $\tilde{3}: \tilde{1}$ and by $(\tilde{2}: \tilde{1})-(\tilde{4}: \tilde{1})$. It follows that $\xi(\tilde{3}: \tilde{1})=$ $\left\{1, \frac{1}{3}\right\}_{14}$ and $\xi(\tilde{2}: \tilde{1})-\xi(\tilde{4}: \tilde{1})=\left\{1, \frac{1}{2}\right\}_{14}-\left\{1, \frac{1}{4}\right\}_{14}$ form a base of $H_{1}(X(14), Z)$.

4. In the following tables the line

$$
N(N=11 \text { or } 14)
$$

$n$\begin{tabular}{|c|c|c|}
\hline$\pm\left(x_{1}, x_{2}, x_{3}\right)$ & $t+u \sqrt{d}$ & $p_{0}+\left[p_{1}, \cdots, p_{k}\right]$ \\
\hline$\pm\left(y_{1}, y_{2}, y_{3}\right)$ & $\prime \prime$ & $q_{0}+\left[q_{1}, \cdots, q_{l}\right]$ \\
\hline
\end{tabular}

means that $\pm\left(x_{1} X^{2}+x_{2} X Y+x_{3} Y^{2}\right)$ and $\pm\left(y_{1} X^{2}+y_{2} X Y+y_{3} Y^{2}\right)$ form a complete set of representatives of binary quadratic forms in $L(N)^{(9)}$ with discriminant $d=N n$ (resp. $d=4 n N$ ) for $N=11$ (resp. 14); that $\varepsilon_{d}$ which was introduced in Lemma 2.4 is $t+u \sqrt{d}$; that $\left(2 x_{1} u\right) /\left(t+x_{2} u\right)$ (resp. $\left.\left(2 y_{1} u\right) /\left(t+y_{2} u\right)\right)$ is equal to

$$
p_{0}+\frac{1}{p_{1}}+\frac{1}{p_{2}}+\cdots+\frac{1}{p_{k}}\left(\text { resp. } q_{0}+\frac{1}{q_{0}}+\cdots+\frac{1}{q_{\ell}}\right) .
$$

(9) For the definition of $L(N)$, see (2.23). 
TABLE I

$$
N=11
$$

\begin{tabular}{|l|l|l|l|}
\hline$n$ & & & \\
\hline 3 & $\pm(1,11,22)$ & $23+4 \sqrt{33}$ & {$[8,2,1,2]$} \\
\hline 4 & $\pm(1,0,-11)$ & $10+3 \sqrt{11}$ & {$[3,3]$} \\
\hline 7 & $\pm(1,11,11)$ & $(9+\sqrt{77}) / 2$ & {$[10]$} \\
\hline 8 & $\pm(1,0,-22)$ & $197+21 \sqrt{88}$ & {$[4,1,2,4,3]$} \\
\hline
\end{tabular}

\section{TABLE II}

$$
N=14
$$

\begin{tabular}{|c|c|c|c|}
\hline \multicolumn{4}{|l|}{$n$} \\
\hline 1 & $\pm(1,0,-14)$ & $15+2 \sqrt{56}$ & {$[3,1,3]$} \\
\hline 2 & $\pm(1,0,-28)$ & $127+12 \sqrt{112}$ & {$[5,3,2,3]$} \\
\hline \multirow{2}{*}{$3\}$} & $\pm(1,0,-42)$ & $13+\sqrt{168}$ & {$[6,2]$} \\
\hline & $\pm(3,0,-14)$ & $\prime \prime$ & {$[2,6]$} \\
\hline \multirow{2}{*}{5\{} & $\pm(1,0,-70)$ & $251+30 \sqrt{70}$ & {$[8,2,1,2,1,2]$} \\
\hline & $\pm(3,28,42)$ & $\prime \prime$ & {$[7,2,5,8]$} \\
\hline \multirow{2}{*}{6\{} & $\pm(1,0,-84)$ & $55+3 \sqrt{336}$ & {$[9,6]$} \\
\hline & $\pm(5,56,140)$ & "I & {$[7,2,3,4]$} \\
\hline 7 & $\pm(1,0,-98)$ & $99+5 \sqrt{392}$ & {$[9,1,9]$} \\
\hline \multirow{2}{*}{10\{} & $\pm(1,0,-140)$ & $71+3 \sqrt{560}$ & {$[11,1,5]$} \\
\hline & $\pm(13,-168,532)$ & $\prime \prime$ & $-1+[1,4,1,1,4.2,1,2]$ \\
\hline \multirow{2}{*}{11\{} & $\pm(1,0,-154)$ & $21295+858 \sqrt{616}$ & {$[12,2,2,3,1,2,1,3,2,2]$} \\
\hline & $\pm(3,28,14)$ & "1 & {$[8,1,4,12,4,1,7,2]$} \\
\hline
\end{tabular}

4. LEMMA 3.2. Let $\chi^{\prime}$ be a character modulo $4 N$ which satisfies $\chi^{\prime 4}=1$ and let $\nu_{\infty}$ be the number of inequivalent cusps of $\Gamma_{0}(4 N)$. Take $a \psi \in \mathfrak{S}_{3}\left(4 N, \chi^{\prime}\right)$ and set $\psi=\sum_{n=1}^{\infty} a_{n} e[n z]$. Assume $a_{1}=a_{2}=\cdots=a_{n_{0}}=0$ for an integer $n_{0}$ and assume $4 n_{0}$ is larger than $3 \sum_{\delta} N_{\varphi}((\delta, N / \delta)) / \delta(\delta, N / \delta)-$ $\nu_{\infty}+1$, where $\varphi$ is the Euler function and the summation is taken over all positive odd divisors of $N$. Then $\psi$ vanishes identically. 
Proof. A set of representatives for all the inequivalent cusps of $\Gamma_{0}(4 N)$ is given by $\left\{u / \delta ; 0<\delta \mid 4 N,(u, \delta)=1\right.$, $u$ modulo $\left.\left(\delta, 4 N \delta^{-1}\right)\right\}$. For each $u / \delta$, take integers $u^{\prime}, \delta^{\prime}$ which satisfy $u \delta^{\prime}-u^{\prime} \delta=1$. A uniformizing parameter for $\Gamma_{0}(4 N) \backslash \mathfrak{F}_{\mathcal{C}} \cup i \infty \cup \boldsymbol{Q}$ at $u / \delta$ is given by

$$
t=\exp \frac{2 \pi i}{e}\left(\frac{\delta^{\prime} z-u^{\prime}}{-\delta z+u}\right), \text { where } e=4 N / \delta\left(\delta, 4 N \delta^{-1}\right) .
$$

The function $\theta^{12}(z)$ is a regular holomorphic form of weight 6 with respect to $\Gamma_{0}(4 N)$. It has no zeros on $\mathfrak{S}_{2}$. The cusp $u / \delta$ is not a zero of $\theta^{12}$ if $\delta$ is either odd or divisible by 4 . If $\delta$ is even but is not divisible by 4 , $\theta^{12}(z)$ has a zero of order $3 e$ at $u / \delta$, with respect to the parameter $t$. Hence, a meromorphic function $\psi^{4}(z) / \theta^{12}(z)$ on $X(4 N)$ is regular except at cusps $u / \delta$ with $\delta$ even but not divisible by 4 . At $u / 2 \delta_{0}\left(\delta_{0}\right.$ odd, $\left.\delta_{0} \mid N\right)$, $\psi^{4}(z) / \theta^{12}(z)$ has a pole of order at most $3 e-1=3 N / \delta_{0}\left(\delta_{0}, N \delta_{0}^{-1}\right)-1$. Since $\psi^{4}(z)$ vanishes at all cusps and has a zero of order at least $4 n_{0}$ at $1 / 4 N$, we have

$$
4 n_{0}+\nu_{\infty}-1 \leqslant 3 \sum_{\delta_{0} \text { odd, } \delta_{0} \mid N}\left\{N / \delta_{0}\left(\delta_{0}, N \delta_{0}^{-1}\right)\right\} \varphi\left(\delta_{0}, N \delta_{0}^{-1}\right)
$$

if $\psi$ dose not vanish identically. The lemma is now proved.

5. Set $N=11, \chi(x)=\left(\frac{x}{11}\right)$. In this case, $S_{2}\left(\Gamma_{0}(11), 1\right)$ is onedimensional and is spanned by $\varphi(z)={ }^{12} \sqrt{\Delta(z) \Delta(11 z)}$, where $\Delta(z)$ is given by $(3,1)$. Further $\Theta_{x}(z, \varphi) \in \mathbb{S}_{3}(44,1)$. Let $\bigcirc$ be the maximal order of $Q(\sqrt{-11})$. For each $x \in \mathfrak{D}$, let $\nu(x)$ be 0 or $\rho=(-1+\sqrt{-3}) / 2$ or $\rho^{2}$ or 1 according as $x$ is congruent modulo 2 to 0 or $(1+\sqrt{-11}) / 2$ or $(-1+\sqrt{-11}) / 2$ or 1 . Set

$$
\begin{aligned}
f(z) & =2^{-1} \sum_{x \in 0} \nu(x) e[z N(x)] \quad(N(x) \text { is the norm of } x) \\
& =q-q^{3}-q^{5}+q^{11}+q^{15}-q^{23}+q^{27}-q^{31}-q^{33}+\cdots \\
& =q \prod_{n=1}^{\infty}\left(1-q^{2 n}\right)\left(1-q^{22 n}\right) \quad(q=e[z]) .
\end{aligned}
$$

Then $f(z) \in S_{1}\left(44, \chi^{\prime \prime}\right)$, where $\chi^{\prime \prime}$ is a character given by $\chi^{\prime \prime}(d)=\left(\frac{-44}{d}\right)$. Further, set

$$
\begin{aligned}
\theta(11 z) f(z)= & q-q^{3}-q^{5}+q^{11}+2 q^{12}-2 q^{14}+q^{15}-2 q^{16}+2 q^{22} \\
& -q^{23}+2 q^{26}+q^{27}-q^{31}-q^{33}+\cdots \\
= & \sum_{n \geq 1} a_{n} q^{n}
\end{aligned}
$$


We have $\theta(11 z) f(z) \in \mathfrak{S}_{3}(44,1)$. Hence, by Theorem 1.7 of [10],

$$
\Im_{3}(44,1) \ni\left(f \mid T_{3,1}^{44}\left(2^{2}\right)\right)(z)=\sum_{n \geq 1} a_{4 n} q^{n}=2 q^{3}-2 q^{4}-2 q^{11} \cdots .
$$

Set $\gamma_{1}=\left\{0, \frac{1}{2}\right\}_{11}$ and $\gamma_{2}=\left\{0, \frac{1}{3}\right\}_{11}$ (for notations, see 3 ). We have seen, in (i) of examples of Lemma 3, that $\gamma_{1}$ and $\gamma_{2}$ form a base of $H_{1}(X(11), Z)$. Put $\omega_{1}=\int_{\gamma_{1}} \varphi(z) d z$ and $\omega_{2}=\int_{r_{2}} \varphi(z) d z$. Then, $\omega_{1}$ and $\omega_{2}$ are linearly independent over $\boldsymbol{R}$.

Proposition 3.3.

$$
\Theta_{\chi}(z, \varphi)=\left(\omega_{1}-2 \omega_{2}\right) \sum_{n \geq 1} a_{4 n} e[n z],
$$

where $\sum_{n \geq 1} a_{n} e[n z]$ is given by (3.11).

Proof. Set $\Theta_{x}(z, \varphi)=\sum_{n \geq 1} b_{n} e[n z]$. Making use of (3.5), Lemma 3.1 and Table I, we have after straightforward computations $b_{1}=b_{2}=0$, $b_{3}=2\left(\omega_{1}-2 \omega_{2}\right), b_{4}=2\left(2 \omega_{2}-\omega_{1}\right), b_{5}=b_{6}=b_{7}=b_{8}=0$. Hence, $\Theta_{x}(z, \varphi)-$ $\left(\omega_{1}-2 \omega_{2}\right) \sum_{n \geq 1} a_{4 n} e[n z]$ has a zero at $i \infty$ of order at least eight. Since both sides of $(3.12)$ belong to $\widetilde{\Im}_{3}(44,1)$, it follows from Lemma 3.2 that both sides of (3.12) coincide.

Remark 3.1. We have,

$$
b_{11}=\sum_{x \text { mod.11 }}\left(\frac{x}{11}\right) \int_{i \infty}^{x / 11} \varphi(z) d z=\sqrt{11} D_{x}^{\varphi}(1),
$$

where

$$
D_{\chi}^{\varphi}(s)=(2 \pi)^{-s} \Gamma(s) \sum_{n \geq 1}\left(\frac{\alpha_{n}}{n^{s}}\right)\left(\frac{n}{11}\right) \quad\left(\varphi(z)=\sum_{n \geq 1} \alpha_{n} q^{n}\right)
$$

6. Set $N=14, \chi(x)=\left(\frac{x}{7}\right)$ (we understand that $\chi$ is a character modulo 14). In this case $S_{2}\left(\Gamma_{0}(14), 1\right)$ is one-dimensional and is spanned by

$$
\begin{aligned}
\varphi(z) & ={ }^{24} \sqrt{\Delta(z) \Delta(2 z) \Delta(7 z) \Delta(14 z)} \\
& =q \prod_{n=1}^{\infty}\left(1-q^{n}\right)\left(1-q^{2 n}\right)\left(1-q^{7 n}\right)\left(1-q^{14 n}\right) \\
& =q-q^{2}-2 q^{3} \cdots \quad(q=e[z]) .
\end{aligned}
$$

By (3.7), $\Theta_{\chi}(z, \varphi) \in \Im_{3}\left(56, \chi^{\prime}\right)$, where $\chi^{\prime}$ is a character modulo 56 given by $\chi^{\prime}(d)=\left(\frac{2}{d}\right)$. Set 


$$
\begin{aligned}
f(z) & =\frac{1}{2} \sum_{(x, y) \in Z^{2}}\left\{e\left[z\left(x^{2}+14 y^{2}\right)\right]-e\left[z\left(2 x^{2}+7 y^{2}\right)\right]\right\} \\
& =q-q^{2}+q^{4}-q^{7}-q^{8}-q^{9}+q^{14}+q^{16}+q^{18}+2 q^{23} \ldots .
\end{aligned}
$$

Then $f(z) \in S_{1}\left(56, \chi^{\prime \prime}\right)$, where $\chi^{\prime \prime}$ is a character modulo 56 given by $\chi^{\prime \prime}(d)$ $=\left(\frac{-56}{d}\right)$. Put

$$
\begin{aligned}
\theta(7 z) f(z) & =\sum_{n \geq 1} a_{n} q^{n} \\
& =q-q^{2}+q^{4}-q^{7}+q^{8}-3 q^{9}+2 q^{11} \ldots
\end{aligned}
$$

Then $\theta(7 z) f(z) \in \Im_{3}\left(56, \chi^{\prime}\right)$. Furthermore

$$
\sum_{n \geq 1} a_{4 n} q^{n}=q+q^{2}-q^{4}-q^{7}-q^{8}-3 q^{9}+2 q^{11}+\cdots
$$

is also a member of $\Im_{3}\left(56, \chi^{\prime}\right)$. Set $\gamma_{1}=\left\{1, \frac{1}{3}\right\}_{14}$ and $\gamma_{2}=\left\{1, \frac{1}{2}\right\}_{14}-\left\{1, \frac{1}{4}\right\}_{14}$ (for notations see $3^{\circ}$ ). We have seen, in (ii) of examples of Lemma 3.1, that $\gamma_{1}$ and $\gamma_{2}$ form a base of $H_{1}(X(14), Z)$. Put $\omega_{1}=\int_{\gamma_{1}} \varphi(z) d z, \omega_{2}=$ $\int_{\gamma_{2}} \varphi(z) d z$. Then $\omega_{1}$ and $\omega_{2}$ are linearly independent over $\boldsymbol{R}$.

Proposition 3.4.

$$
\Theta_{\chi}(z, \varphi)=2\left(\omega_{1}-\omega_{2}\right) \sum_{n \geq 1} a_{4 n} q^{n}
$$

Proof. Set

$$
\Theta_{\chi}(z, \varphi)=\sum_{n \geq 1} b_{n} q^{n}
$$

Making use of (3.6), Lemma 3.1 and Table II, we have, after some computations, $b_{1}=2\left(\omega_{1}-\omega_{2}\right), b_{2}=2\left(\omega_{1}-\omega_{2}\right), b_{3}=b_{5}=b_{6}=0, b_{7}=2\left(\omega_{2}-\omega_{1}\right)$, $b_{10}=0$ and $b_{11}=4\left(\omega_{1}-\omega_{2}\right)$.

Since $S_{2}\left(\Gamma_{0}(14), 1\right)$ is one-dimensional, it follows from (2.18) and (3.13) that $T_{2}^{14}\left(2, \chi^{2}\right) \varphi=-\varphi$ and $T_{2}^{14}\left(3, \chi^{2}\right) \varphi=-2 \varphi$. Hence, by Theorem $2, b_{4}=$ $-b_{1}, b_{8}=-b_{2}$ and $-2 b_{1}=b_{9}+b_{1}$. Thus, we see, by (3.14) that $\Theta_{\chi}(z, \varphi)-2\left(\omega_{1}-\omega_{2}\right) \sum_{n \geq 1} a_{4 n} q^{n}$ has a zero at $i \infty$ of order at least 11 . Since $\Theta_{\chi}(z, \varphi)$ and $2\left(\omega_{1}-\omega_{2}\right) \sum_{n \geq 1} a_{4 n} q^{n}$ both belong to $\widetilde{S}_{3}\left(56, \chi^{\prime}\right)$, we have $\Theta_{x}(z, \varphi)=2\left(\omega_{1}-\omega_{2}\right) \sum_{n \geq 1} a_{4 n} q^{n}$, by Lemma 3.2.

Remark (i) If $\chi(-1)=+1$, then the integral (3.5) and (3.6) vanish identically. Since $\chi(-1)=1$ for any quadratic character modulo 17, (3.5) vanishes if $N=17$. 


\section{REFERENCES}

[1] Dirichlets-Dedekind: Vorlesungen über die Zahlentheorie.

[2] I. Gelfand-I. Graev-Pyatetskii-Shapiro: Representation theory and automorphic functions, Saunders Company.

[ 3 ] R. Godement: Introduction à la théorie de Langlands, Séminaire Bourbaki, Exposé $321,1966$.

[4] E. Hecke: Algebraische Zahlen, Chelsea.

[ 5 ] J. Igusa: Theta functions, Springer Verlag.

[6] J. Manin: Parabolic points and zeta-functions of modular curves, Izv. Akad. Nauk, SSSR, Ser. Mat. 36 (1972), 19-66.

[7] H. Maass: Über eine neue Art von nichtanalytischen automorphen Funktionen und die Bestimmung Dirichletscher Reihen durch ihre Funktionalgleichung, Math. Ann. 121 (1949), 141-183.

[ 8 ] — : Über die räumliche Verteilung der Punkte in Gittern mit indefiniter Metrik, Math. Ann. 138 (1959), 287-315.

[ 9 ] J. A. Shalika and S. Tanaka: On an explicit construction of a certain class of automorphic forms, Amer. J. Math. 91 (1969), 1049-1076.

[10] G. Shimura: On modular forms of half integral weight, Ann. of Math. 97 (1973), $440-481$.

[11] _ - Introduction to the arithmetic theory of automorphei functions, Publ. Math. Soc. Japan, $\mathrm{n}^{\circ} 11$.

[12] C. L. Siegel: Indefinite quadratische Formen und Funktionen Theorie I, Math. Ann. 124 (1951), 17-54; II, Math. Ann. 124 (1952), 364-387.

[13] André Weil: Sur certains groupes d'opérateurs unitaires, Acta Math. 111 (1964), 143-211.

[14] Manin: Periods of parabolic forms and p-adic Hecke series, (Russian) Mat. Sb. 92 (1973), 378-401.

Department of Mathematics

Faculty of Science

University of Tokyo 